\title{
Projective Metric Geometry and Clifford Algebras
}

\author{
Hans Havlicek
}

Dedicated to the memory of Heinrich Wefelscheid

\begin{abstract}
Each vector space that is endowed with a quadratic form determines its Clifford algebra. This algebra, in turn, contains a distinguished group, known as the Lipschitz group. We show that only a quotient of this group remains meaningful in the context of projective metric geometry. This quotient of the Lipschitz group can be viewed as a point set in the projective space on the Clifford algebra and, under certain restrictions, leads to an algebraic description of so-called kinematic mappings.
\end{abstract}

Mathematics Subject Classification. 51F25, 15A66, 51F15.

Keywords. projective metric space, Clifford algebra, Lipschitz monoid, Lipschitz group, kinematic mapping.

\section{Introduction}

By a metric vector space we mean a finite dimensional vector space $\boldsymbol{V}$ (over a field $F$ of arbitrary characteristic) that is endowed with a quadratic form $Q$. The description of orthogonal transformations of a metric vector space $(\boldsymbol{V}, Q)$ in terms of its associated Clifford algebra $\mathrm{Cl}(\boldsymbol{V}, Q)$ has a long history. We follow the exposition by Schröder [50] and provide in Sect. 2 basic facts about a metric vector space $(\boldsymbol{V}, Q)$ and its weak orthogonal group $\mathrm{O}^{\prime}(\boldsymbol{V}, Q)$, which in most cases is generated by reflections. In Sect. 3, we collect from various sources those results about the Clifford algebra $\mathrm{Cl}(\boldsymbol{V}, Q)$ which are needed later on. Section 4 is based on the work of Helmstetter as summarised in [25]. We recall from there the $\operatorname{Lipschitz}$ monoid $\operatorname{Lip}(\boldsymbol{V}, Q)$ and the twisted adjoint 
representation of the Lipschitz group $\operatorname{Lip}^{\times}(\boldsymbol{V}, Q)$, which provides a surjective homomorphism onto the weak orthogonal group $\mathrm{O}^{\prime}(\boldsymbol{V}, Q)$.

The main goal of the present note is the interpretation of the Lipschitz group $\operatorname{Lip}^{\times}(\boldsymbol{V}, Q)$ in projective terms, that is, we consider the projective metric space $\mathbb{P}(\boldsymbol{V}, Q)$ and the projective space on the associated Clifford algebra $\mathrm{Cl}(\boldsymbol{V}, Q)$. Thereby one is immediately facing the following problem: if the quadratic form $Q$ is replaced by a non-zero multiple, say $c Q$ with $c \in F \backslash\{0\}$, then this does not affect the geometry of $\mathbb{P}(\boldsymbol{V}, Q)$, but the Clifford algebras $\mathrm{Cl}(\boldsymbol{V}, Q)$ and $\mathrm{Cl}(\boldsymbol{V}, c Q)$ need not be isomorphic. Therefore, the usage of Clifford algebras in projective metric geometry at a first sight appears to be problematic.

We start Sect. 5 by introducing in $\mathbb{P}(\mathrm{Cl}(\boldsymbol{V}, Q))$ point sets $\mathcal{M}(\boldsymbol{V}, Q)$ and $\mathcal{G}(\boldsymbol{V}, Q)$ that arise from a quotient of the $\operatorname{Lipschitz} \operatorname{monoid} \operatorname{Lip}(\boldsymbol{V}, Q)$ and a quotient of the Lipschitz group $\operatorname{Lip}^{\times}(\boldsymbol{V}, Q)$. The latter set can be made into a group in a natural way and as such it acts on the initial projective metric space $\mathbb{P}(\boldsymbol{V}, Q)$. In Theorems $5.4,5.5$ and 5.6 we carry out a detailed study of this group action and its kernel, thereby extending previous work of Gunn [19,20], Jurk [32], Klawitter and Hagemann [36], Klawitter [35], Schröder [49] and others. Since the details are somewhat involved, an alternative point of view is adopted in Tables 1, 2 and 3. These tables allow us to read off all those instances, where a kind of "kinematic mapping" for the projective weak orthogonal group $\mathrm{PO}^{\prime}(\boldsymbol{V}, Q)$ can be obtained. Next, in Sect. 6, we return to the problem sketched above by comparing the Clifford algebras $\mathrm{Cl}(\boldsymbol{V}, Q)$ and $\mathrm{Cl}(\boldsymbol{V}, c Q)$. From a result by Knus [40, Ch. IV, (7.1.1)], we are in a position to identify the underlying vector spaces of these algebras in such a way that, firstly, their even subalgebras $\mathrm{Cl}_{0}(\boldsymbol{V}, Q)$ and $\mathrm{Cl}_{0}(\boldsymbol{V}, c Q)$ coincide (as algebras), secondly, their odd parts $\mathrm{Cl}_{1}(\boldsymbol{V}, Q)$ and $\mathrm{Cl}_{1}(\boldsymbol{V}, c Q)$ are the same (as vector subspaces), thirdly, the two multiplications are related in a manageable way. Using this identification, it turns out that all our results from Sect. 5 remain unaltered when going over from $Q$ to $c Q$. This is due to the fact that the Clifford algebra $\mathrm{Cl}(\boldsymbol{V}, Q)$ just serves as a kind of "container" for its even and odd part, but we never use any element of $\mathrm{Cl}(\boldsymbol{V}, Q)$ from outside these two subspaces. Finally, Sect. 7 provides a list of open questions that may lead to future research.

Let us close by pointing out that our note is not intended to be a critical survey. We therefore mainly quote such work that will clear the way to previous contributions. Also, whenever we just refer to other sources without using them, we usually do not emphasise diverging definitions, differing hypotheses and other deviations from our approach.

\section{Metric Vector Spaces}

Let $\boldsymbol{V}$ be a vector space with finite dimension $n+1 \geq 0$ over a (commutative) field $F$. We suppose that $\boldsymbol{V}$ is equipped with a quadratic form $Q: \boldsymbol{V} \rightarrow F$; the 
zero form is not excluded. There is a widespread literature about quadratic forms; see, for example, [8, Ch. 8], [13,43] or [53]. We adopt the terminology from [50] by addressing $(\boldsymbol{V}, Q)$ as a metric vector space. A non-zero vector $\boldsymbol{a} \in \boldsymbol{V}$ is called regular if $Q(\boldsymbol{a}) \neq 0$ and singular otherwise. Observe that none of these attributes applies to the zero vector. A subspace of $\boldsymbol{V}$ is said to be totally singular if all its non-zero vectors are singular.

Let $B: \boldsymbol{V} \times \boldsymbol{V} \rightarrow F:(\boldsymbol{x}, \boldsymbol{y}) \mapsto Q(\boldsymbol{x}+\boldsymbol{y})-Q(\boldsymbol{x})-Q(\boldsymbol{y})$ denote the polar form of $Q$, which is a symmetric bilinear form. Then, for all $\boldsymbol{x} \in \boldsymbol{V}$, $B(\boldsymbol{x}, \boldsymbol{x})=2 Q(\boldsymbol{x})$. Orthogonality is written as $\perp$; that is, for all $\boldsymbol{x}, \boldsymbol{y} \in \boldsymbol{V}$, $\boldsymbol{x} \perp \boldsymbol{y}$ means $B(\boldsymbol{x}, \boldsymbol{y})=0$. Each subset $\boldsymbol{S} \subseteq \boldsymbol{V}$ determines the subspace $\boldsymbol{S}^{\perp}:=\{\boldsymbol{x} \in \boldsymbol{V} \mid \boldsymbol{x} \perp \boldsymbol{y}$ for all $\boldsymbol{y} \in \boldsymbol{S}\}$ of $\boldsymbol{V}$. In particular, $\boldsymbol{V}^{\perp}$ is called the radical of $B$. The form $B$ is said to be non-degenerate provided that $\boldsymbol{V}^{\perp}=\{0\}$.

Let $(\tilde{\boldsymbol{V}}, \tilde{Q})$ also be a metric vector space over $F$. A linear bijection $\psi: \boldsymbol{V} \rightarrow \tilde{\boldsymbol{V}}$ is called a similarity if $c Q=\tilde{Q} \circ \psi$ for some $c \in F^{\times}:=F \backslash\{0\}$. Provided that $Q(\boldsymbol{V}) \neq\{0\}$, the scalar $c$ is uniquely determined by $\psi$ and it will be addressed as the ratio of $\psi$. Whenever $Q(\boldsymbol{V})=\{0\}$ we adopt the convention to consider only $1 \in F^{\times}$as being the ratio of $\psi$. An isometry is understood to be a similarity of ratio $c=1$.

We recall that the general orthogonal group $\operatorname{GO}(\boldsymbol{V}, Q)$ is that subgroup of the general linear group $\mathrm{GL}(\boldsymbol{V})$ which comprises all similarities of $(\boldsymbol{V}, Q)$ onto itself. All isometries of $(\boldsymbol{V}, Q)$ onto itself constitute the orthogonal group $\mathrm{O}(\boldsymbol{V}, Q)$. The weak orthogonal group ${ }^{1} \mathrm{O}^{\prime}(\boldsymbol{V}, Q)$ consists of all isometries of $(\boldsymbol{V}, Q)$ that fix the radical $\boldsymbol{V}^{\perp}$ elementwise. Each regular vector $\boldsymbol{r} \in \boldsymbol{V}$ determines the mapping

$$
\xi_{r}: \boldsymbol{V} \rightarrow \boldsymbol{V}: \boldsymbol{x} \mapsto \boldsymbol{x}-B(\boldsymbol{r}, \boldsymbol{x}) Q(\boldsymbol{r})^{-1} \boldsymbol{r} .
$$

We call $\xi_{\boldsymbol{r}}$ the reflection of $(\boldsymbol{V}, Q)$ in the direction of $\boldsymbol{r}$ and note that $\xi_{\boldsymbol{r}} \in$ $\mathrm{O}^{\prime}(\boldsymbol{V}, Q)$. Under $\xi_{\boldsymbol{r}}$ all vectors in $\boldsymbol{r}^{\perp}$ are fixed and $\boldsymbol{r}$ goes over to $\boldsymbol{- r}$. Also, $\xi_{\boldsymbol{r}}$ is the identity on $\boldsymbol{V}$ if, and only if, $\boldsymbol{r}$ is a regular vector in the radical $\boldsymbol{V}^{\perp}$; this can only happen in case of characteristic 2 ; see $[50,1.6 .2]$. We are now in a position to write up a version of the classical Cartan-Dieudonné Theorem as follows. Each isometry $\phi \in \mathrm{O}^{\prime}(\boldsymbol{V}, Q)$ is a product of reflections, except when $F$ and $(\boldsymbol{V}, Q)$ satisfy one of the subsequent conditions (2) or (3) for some basis $\left\{\boldsymbol{e}_{0}, \boldsymbol{e}_{1}, \ldots, \boldsymbol{e}_{n}\right\}$ of $\boldsymbol{V}$ and all $\boldsymbol{x}=\sum_{j=0}^{n} x_{j} \boldsymbol{e}_{j}$ with $x_{j} \in F$ :

$$
\begin{aligned}
& |F|=2, \operatorname{dim} \boldsymbol{V} \geq 2 \text { and } Q(\boldsymbol{x})=x_{0} x_{1} ; \\
& |F|=2, \operatorname{dim} \boldsymbol{V}=4 \text { and } Q(\boldsymbol{x})=x_{0} x_{1}+x_{2} x_{3} .
\end{aligned}
$$

We refer to $[15,16,39]$ for proofs and to $[4,9-12,14,29,37],[43$, p. 18], [46], [50, 1.6.3], [53, pp. 156-159] for further details, generalisations and additional references. Let us just mention that the reflections of $(\boldsymbol{V}, Q)$ generate a proper

\footnotetext{
${ }^{1}$ We follow here the terminology and notation from [9]. In German this group is known under the name "orthogonale Gruppe im engeren Sinne". $\mathrm{O}^{\prime}(\boldsymbol{V}, Q)$ must not be confused with the derived group of $\mathrm{O}(\boldsymbol{V}, Q)$; see [8, p. 39].
} 
subgroup of its weak orthogonal group whenever $F$ and $(\boldsymbol{V}, Q)$ meet the requirements of (2) or (3).

\section{Clifford Algebras}

Let $(\boldsymbol{V}, Q)$ be a metric vector space over $F$ (as in $\operatorname{Sect.} 2$ ) and let $\operatorname{Cl}(\boldsymbol{V}, Q)$ denote its Clifford algebra; see, among others, [7, pp. 35ff. and 101ff.], [8, 8.4], [18, Ch. 8, Ch. 13], [31, Ch. 3] [40, Ch. IV] or [43, Ch. 5]. In our study we shall adopt two widely used conventions. Firstly, we identify $1 \in F$ with the unit element of the $F$-algebra $\mathrm{Cl}(\boldsymbol{V}, Q)$ and, secondly, we consider $\boldsymbol{V}$ as being a subspace of $\mathrm{Cl}(\boldsymbol{V}, Q)$. So $\mathrm{Cl}(\boldsymbol{V}, Q)$ is the universal associative and unital algebra over $F$ that is generated by $\boldsymbol{V}$ and subject to the relations $Q(\boldsymbol{x})=\boldsymbol{x}^{2}$ for all $\boldsymbol{x} \in \boldsymbol{V}$. Consequently, for all $\boldsymbol{x}, \boldsymbol{y} \in \boldsymbol{V}$, we have $B(\boldsymbol{x}, \boldsymbol{y})=\boldsymbol{x} \boldsymbol{y}+\boldsymbol{y} \boldsymbol{x}$. We now write up some well known properties of $\mathrm{Cl}(\boldsymbol{V}, Q)$ in order to fix our notation.

The Clifford algebra $\mathrm{Cl}(\boldsymbol{V}, Q)$ is $\mathbb{Z} /(2 \mathbb{Z})$-graded and so it is the direct sum of the even part $\mathrm{Cl}_{0}(\boldsymbol{V}, Q)$, which is a subalgebra of $\mathrm{Cl}(\boldsymbol{V}, Q)$, and the odd part $\mathrm{Cl}_{1}(\boldsymbol{V}, Q)$. If $\boldsymbol{h} \in \mathrm{Cl}_{i}(\boldsymbol{V}, Q), i \in\{0,1\}$, then we say that $\boldsymbol{h}$ is homogeneous of degree $i$ and write $\partial \boldsymbol{h}=i$. Given any subset $\boldsymbol{S} \subseteq \mathrm{Cl}(\boldsymbol{V}, Q)$ we let $\boldsymbol{S}_{i}:=$ $\boldsymbol{S} \cap \mathrm{Cl}_{i}(\boldsymbol{V}, Q)$ for $i \in\{0,1\}$ and we denote by $\boldsymbol{S}^{\times}$the set of all units (w.r.t. multiplication) in $\boldsymbol{S}$.

The main involution $\sigma: \mathrm{Cl}(\boldsymbol{V}, Q) \rightarrow \mathrm{Cl}(\boldsymbol{V}, Q)$ is the only algebra endomorphism of $\mathrm{Cl}(\boldsymbol{V}, Q)$ such that $\boldsymbol{x} \mapsto-\boldsymbol{x}$ for all $\boldsymbol{x} \in \boldsymbol{V}$. Under $\sigma$ all elements of $\mathrm{Cl}_{0}(\boldsymbol{V}, Q)$ remain fixed, any $\boldsymbol{h} \in \mathrm{Cl}_{1}(\boldsymbol{V}, Q)$ goes over to $-\boldsymbol{h}$. The reversal $\alpha: \mathrm{Cl}(\boldsymbol{V}, Q) \rightarrow \mathrm{Cl}(\boldsymbol{V}, Q)$ is the only algebra antiendomorphism of $\mathrm{Cl}(\boldsymbol{V}, Q)$ such that $\boldsymbol{x} \mapsto \boldsymbol{x}$ for all $\boldsymbol{x} \in \boldsymbol{V}$. Each of the mappings $\sigma$ and $\alpha$ is a bijection leaving invariant $\mathrm{Cl}_{0}(\boldsymbol{V}, Q)$ and $\mathrm{Cl}_{1}(\boldsymbol{V}, Q)$.

$\mathrm{Cl}(\boldsymbol{V}, Q)$ is endowed with an (increasing) canonical filtration by subspaces $\mathrm{Cl}^{\leq k}(\boldsymbol{V}, Q), k \in \mathbb{Z}$, as follows [31, pp. 108-109]: if $k<0$ then $\mathrm{Cl}^{\leq k}(\boldsymbol{V}, Q)$ $=\{0\}$; if $k \geq 0$ then $\mathrm{Cl}^{\leq k}(\boldsymbol{V}, Q)$ is that subspace of $\mathrm{Cl}(\boldsymbol{V}, Q)$ which is generated by all products of at most $k$ vectors from $\boldsymbol{V}$. Thereby an empty product of vectors is understood to be $1 \in F \subseteq \mathrm{Cl}(\boldsymbol{V}, Q)$. If $\left\{\boldsymbol{e}_{0}, \boldsymbol{e}_{1}, \ldots, \boldsymbol{e}_{n}\right\}$ is a basis of $\boldsymbol{V}$, then we obtain a basis of $\mathrm{Cl}(\boldsymbol{V}, Q)$ as

$$
\left\{\boldsymbol{e}_{j_{1}} \boldsymbol{e}_{j_{2}} \cdots \boldsymbol{e}_{j_{k}} \mid 0 \leq j_{1}<j_{2}<\cdots<j_{k} \leq n\right\} .
$$

Let $\boldsymbol{T}$ be a subspace of $\boldsymbol{V}$. The restriction $Q \mid \boldsymbol{T}$ makes $\boldsymbol{T}$ into a metric vector space. The unital subalgebra of $\mathrm{Cl}(\boldsymbol{V}, Q)$ generated by $\boldsymbol{T}$ will be considered as the Clifford algebra of $(\boldsymbol{T}, Q \mid \boldsymbol{T})$.

Suppose that $Q(\boldsymbol{V})=\{0\}$. Then $\mathrm{Cl}(\boldsymbol{V}, Q)$ coincides (upon writing " $\wedge$ " instead of ".") with the exterior algebra $\wedge \boldsymbol{V}$. Let $\boldsymbol{m}$ be any element of $\mathrm{Cl}_{1}(\boldsymbol{V}, Q)=\bigoplus_{j \in\{1,3,5, \ldots\}} \bigwedge^{j} \boldsymbol{V}$. From $\bigwedge \boldsymbol{V}$ being $\mathbb{N}$-graded (see [31, p. 185]), the product of $\boldsymbol{m}$ and any $\boldsymbol{n} \in \mathrm{Cl}(\boldsymbol{V}, Q)=\bigwedge \boldsymbol{V}$ belongs to the subspace 
$\bigoplus_{k \in\{1,2,3, \ldots\}} \bigwedge^{k} \boldsymbol{V}$. Therefore $\boldsymbol{m}$ fails to be invertible and we note, for later use:

$$
Q(\boldsymbol{V})=\{0\} \text { implies } \mathrm{Cl}_{1}^{\times}(\boldsymbol{V}, Q)=\emptyset .
$$

The following results are taken from [40, Ch. IV, (7.1.1)] in a form tailored to our needs. Let $(\boldsymbol{V}, Q)$ and $(\tilde{\boldsymbol{V}}, \tilde{Q})$ denote metric vector spaces and suppose that $\psi: \boldsymbol{V} \rightarrow \tilde{\boldsymbol{V}}$ is a similarity of ratio $c \in F^{\times}$. Then there is a unique homomorphism of algebras $\mathrm{Cl}_{0}(\psi): \mathrm{Cl}_{0}(\boldsymbol{V}, Q) \rightarrow \mathrm{Cl}_{0}(\tilde{\boldsymbol{V}}, \tilde{Q})$ such that, for all $\boldsymbol{x}, \boldsymbol{y} \in \boldsymbol{V}$,

$$
\mathrm{Cl}_{0}(\psi)(\boldsymbol{x y})=c^{-1} \psi(\boldsymbol{x}) \psi(\boldsymbol{y}) .
$$

Furthermore, there is a unique linear mapping $\mathrm{Cl}_{1}(\psi): \mathrm{Cl}_{1}(\boldsymbol{V}, Q) \rightarrow \mathrm{Cl}_{1}(\tilde{\boldsymbol{V}}, \tilde{Q})$ such that, for all $\boldsymbol{p} \in \mathrm{Cl}_{0}(\boldsymbol{V}, Q)$ and all $\boldsymbol{x} \in \boldsymbol{V}$,

$$
\begin{aligned}
& \mathrm{Cl}_{1}(\psi)(\boldsymbol{p} \boldsymbol{x})=\mathrm{Cl}_{0}(\psi)(\boldsymbol{p}) \cdot \psi(\boldsymbol{x}), \\
& \mathrm{Cl}_{1}(\psi)(\boldsymbol{x p})=\psi(\boldsymbol{x}) \cdot \mathrm{Cl}_{0}(\psi)(\boldsymbol{p}) .
\end{aligned}
$$

Take notice that, for all $\boldsymbol{x} \in \boldsymbol{V}, \mathrm{Cl}_{1}(\psi)(\boldsymbol{x})=\psi(\boldsymbol{x})$ follows from (7) by letting $\boldsymbol{p}=1$. This motivates our name Clifford extension of $\psi$ for the mapping

$$
\mathrm{Cl}_{0}(\psi) \oplus \mathrm{Cl}_{1}(\psi)=: \mathrm{Cl}(\psi): \mathrm{Cl}(\boldsymbol{V}, Q) \rightarrow \mathrm{Cl}(\tilde{\boldsymbol{V}}, \tilde{Q}) .
$$

Since $\psi^{-1}$ is a similarity of ratio $c^{-1}$, there exists $\mathrm{Cl}\left(\psi^{-1}\right)=\mathrm{Cl}(\psi)^{-1}$. Thus, by virtue of $\mathrm{Cl}_{0}(\psi)$, the even Clifford algebras $\mathrm{Cl}_{0}(\boldsymbol{V}, Q)$ and $\mathrm{Cl}_{0}(\tilde{\boldsymbol{V}}, \tilde{Q})$ are isomorphic.

Even though the domain of $\mathrm{Cl}(\psi)$ is the entire Clifford algebra $\mathrm{Cl}(\boldsymbol{V}, Q)$, we shall predominantly apply this mapping to homogeneous elements of $\mathrm{Cl}(\boldsymbol{V}, Q)$. In particular, the following formula will turn out crucial, as it describes to which extent $\mathrm{Cl}(\psi)$ "deviates" from an isomorphism of algebras. Given homogeneous elements $\boldsymbol{m}, \boldsymbol{n} \in \mathrm{Cl}(\boldsymbol{V}, Q)$ we assert that

$$
\mathrm{Cl}(\psi)(\boldsymbol{m n})=c^{-\partial \boldsymbol{m} \partial \boldsymbol{n}} \mathrm{Cl}(\psi)(\boldsymbol{m}) \cdot \mathrm{Cl}(\psi)(\boldsymbol{n}) .
$$

By the additivity of $\mathrm{Cl}(\psi)$ and the law of distributivity, it suffices to verify (9) when $\boldsymbol{m}=\boldsymbol{a}_{1} \boldsymbol{a}_{2} \cdots \boldsymbol{a}_{r}$ and $\boldsymbol{n}=\boldsymbol{a}_{r+1} \boldsymbol{a}_{r+2} \cdots \boldsymbol{a}_{r+s}$ with $\boldsymbol{a}_{1}, \boldsymbol{a}_{2}, \ldots, \boldsymbol{a}_{r+s} \in \boldsymbol{V}$ and $r, s \geq 0$. There are four cases:

Case $1 r$ and $s$ are even. Here (9) holds trivially, since $\mathrm{Cl}_{0}(\psi)$ is a homomorphism of algebras.

Case $2 r$ is even and $s$ is odd. By the first equation in (7) and Case 1,

$$
\begin{aligned}
\mathrm{Cl}_{1}(\psi)(\boldsymbol{m n}) & =\mathrm{Cl}_{1}(\psi)\left(\boldsymbol{m}\left(\boldsymbol{a}_{r+1} \boldsymbol{a}_{r+2} \cdots \boldsymbol{a}_{r+s-1}\right) \boldsymbol{a}_{r+s}\right) \\
& =\mathrm{Cl}_{0}(\psi)\left(\boldsymbol{m} \boldsymbol{a}_{r+1} \boldsymbol{a}_{r+2} \cdots \boldsymbol{a}_{r+s-1}\right) \cdot \psi\left(\boldsymbol{a}_{r+s}\right) \\
& =\mathrm{Cl}_{0}(\psi)(\boldsymbol{m}) \cdot \mathrm{Cl}_{0}(\psi)\left(\boldsymbol{a}_{r+1} \boldsymbol{a}_{r+2} \cdots \boldsymbol{a}_{r+s-1}\right) \cdot \psi\left(\boldsymbol{a}_{r+s}\right) \\
& =\mathrm{Cl}_{0}(\psi)(\boldsymbol{m}) \cdot \mathrm{Cl}_{1}(\psi)(\boldsymbol{n}) .
\end{aligned}
$$

Case $3 r$ is odd and $s$ is even. Writing $\boldsymbol{m}=\boldsymbol{a}_{1}\left(\boldsymbol{a}_{2} \cdots \boldsymbol{a}_{r}\right)$ allows us to proceed in analogy to the previous case, thereby using the second equation in (7). 
Case $4 r$ and $s$ are odd. Now $\boldsymbol{m}=\left(\boldsymbol{a}_{1} \boldsymbol{a}_{2} \cdots \boldsymbol{a}_{r-1}\right) \boldsymbol{a}_{r}$ and $\boldsymbol{n}=\boldsymbol{a}_{r+1}\left(\boldsymbol{a}_{r+2}\right.$ $\left.\cdots \boldsymbol{a}_{r+s}\right)$ together with (6) and (7) establishes (9).

Next, let $\boldsymbol{m}_{1}, \boldsymbol{m}_{2}, \ldots, \boldsymbol{m}_{k}, k \geq 0$, be homogeneous elements of $\mathrm{Cl}(\boldsymbol{V}, Q)$ such that precisely $p$ of them are of degree 1 . Then there is a unique integer $q \geq 0$ with $2 q \leq p \leq 2 q+1$. From (9), we therefore obtain

$$
\mathrm{Cl}(\psi)\left(\boldsymbol{m}_{1} \boldsymbol{m}_{2} \cdots \boldsymbol{m}_{k}\right)=c^{-q} \mathrm{Cl}(\psi)\left(\boldsymbol{m}_{1}\right) \cdot \mathrm{Cl}(\psi)\left(\boldsymbol{m}_{2}\right) \cdots \mathrm{Cl}(\psi)\left(\boldsymbol{m}_{k}\right) .
$$

There are two immediate consequences of (10). Given a homogeneous element $\boldsymbol{m} \in \mathrm{Cl}(\boldsymbol{V}, Q)$ we have

$$
(\mathrm{Cl}(\psi) \circ \alpha)(\boldsymbol{m})=(\tilde{\alpha} \circ \mathrm{Cl}(\psi))(\boldsymbol{m}),
$$

where $\tilde{\alpha}$ denotes the reversal on $\operatorname{Cl}(\tilde{\boldsymbol{V}}, \tilde{Q})$. If, moreover, $\boldsymbol{m}$ is invertible, then

$$
\mathrm{Cl}(\psi)(\boldsymbol{m}) \cdot \mathrm{Cl}(\psi)\left(\boldsymbol{m}^{-1}\right)=c^{\partial \boldsymbol{m}},
$$

which in turn shows that $\mathrm{Cl}(\psi)(\boldsymbol{m})$ is invertible.

\section{Lipschitz Groups}

The following exposition runs along the lines of the survey [25] and the summary in $[31,5.10]$. Historical remarks and additional results may be retrieved from [26-28,30] and [44, pp. 220-230]. According to [25, Def. 2.1] the Lipschitz monoid $\operatorname{Lip}(\boldsymbol{V}, Q)$ is the multiplicative monoid in $\operatorname{Cl}(\boldsymbol{V}, Q)$ generated by the union of $F, \boldsymbol{V}$ and the set

$$
\{1+\boldsymbol{s t} \mid \boldsymbol{s}, \boldsymbol{t} \in \boldsymbol{V}, Q(\boldsymbol{s})=Q(\boldsymbol{t})=B(\boldsymbol{s}, \boldsymbol{t})=0\} .
$$

The Lipschitz monoid $\operatorname{Lip}(\boldsymbol{V}, Q)$ is already generated by $\boldsymbol{V}$ except when one of the following applies: (i) $Q(\boldsymbol{V})=\{0\}$; (ii) $F$ and $(\boldsymbol{V}, Q)$ satisfy (2); (iii) $F$ and $(\boldsymbol{V}, Q)$ satisfy $(3)$; see [23, (7) Thm.], [25, Thm. 2.2].

Three noteworthy properties of any $\boldsymbol{m} \in \operatorname{Lip}(\boldsymbol{V}, Q)$ are as follows [23, (2) Thm.], [25, Thm. 2.7]: (i) $\alpha(\boldsymbol{m}) \in \operatorname{Lip}(\boldsymbol{V}, Q)$; (ii) $\boldsymbol{m} \alpha(\boldsymbol{m})=\alpha(\boldsymbol{m}) \boldsymbol{m} \in F$; (iii) $\boldsymbol{m} \alpha(\boldsymbol{m}) \neq 0$ characterises $\boldsymbol{m}$ as being invertible. Therefore all invertible elements of $\operatorname{Lip}(\boldsymbol{V}, Q)$ constitute a group, the so-called Lipschitz group $\operatorname{Lip}^{\times}(\boldsymbol{V}, Q)$. Furthermore, for all $k \geq 0$ and all $\boldsymbol{z} \in \mathrm{Cl}^{\leq k}(\boldsymbol{V}, Q)$, we have $\boldsymbol{m} \boldsymbol{z} \alpha(\boldsymbol{m}) \in \mathrm{Cl}^{\leq k}(\boldsymbol{V}, Q)$; see [23, (23) Cor.], [25, Thm. 2.8]. This implies, for any $\boldsymbol{m} \in \operatorname{Lip}^{\times}(\boldsymbol{V}, Q)$ and all $\boldsymbol{x} \in \boldsymbol{V}$, that $\boldsymbol{m} \boldsymbol{x} \sigma(\boldsymbol{m})^{-1} \in \boldsymbol{V}$ and that $Q\left(\boldsymbol{m} \boldsymbol{x} \sigma(\boldsymbol{m})^{-1}\right)=Q(\boldsymbol{x})$. The mapping

$$
\xi: \operatorname{Lip}^{\times}(\boldsymbol{V}, Q) \rightarrow \mathrm{O}^{\prime}(\boldsymbol{V}, Q): \boldsymbol{p} \mapsto\left(\xi_{p}: \boldsymbol{x} \mapsto \boldsymbol{p} \boldsymbol{x} \sigma(\boldsymbol{p})^{-1}\right)
$$

is a surjective homomorphism of groups [23, (35) Thm.], [25, Thm. 3.2]; we follow [1] by addressing $\xi$ as the twisted adjoint representation of $\operatorname{Lip}^{\times}(\boldsymbol{V}, Q)$. For any regular vector $\boldsymbol{r} \in \boldsymbol{V}$ we clearly have $\boldsymbol{r} \in \operatorname{Lip}^{\times}(\boldsymbol{V}, Q)$ and the above definition reproduces the reflection $\xi_{r}$ as in (1). 
Remark 4.1. Any element $1+\boldsymbol{s}$ t appearing in (13) is in the Lipschitz group $\operatorname{Lip}^{\times}(\boldsymbol{V}, Q)$, since $(1+\boldsymbol{s} \boldsymbol{t}) \alpha(1+\boldsymbol{s} \boldsymbol{t})=1$. An easy calculation gives, for all $\boldsymbol{x} \in \boldsymbol{V}$,

$$
\xi_{1+s t}(\boldsymbol{x})=\boldsymbol{x}+B(\boldsymbol{t}, \boldsymbol{x}) \boldsymbol{s}-B(\boldsymbol{s}, \boldsymbol{x}) \boldsymbol{t} .
$$

If $\boldsymbol{s}, \boldsymbol{t}$ are linearly dependent, then $\boldsymbol{s t}=0$ and so $\xi_{1+s t}=\mathrm{id}_{\boldsymbol{V}}$. Otherwise, $\xi_{1+s t}$ fixes precisely the vectors of the subspace $\{s, t\}^{\perp}$, which has codimension $\leq 2$ in $\boldsymbol{V}$.

In order to describe the kernel of the twisted adjoint representation (14), we recall the definition of the graded centre of $\mathrm{Cl}(\boldsymbol{V}, Q)$. It is defined as

$$
Z^{g}(\mathrm{Cl}(\boldsymbol{V}, Q)):=Z_{0}^{g}(\mathrm{Cl}(\boldsymbol{V}, Q)) \oplus Z_{1}^{g}(\mathrm{Cl}(\boldsymbol{V}, Q)),
$$

where $Z_{i}^{g}(\mathrm{Cl}(\boldsymbol{V}, Q)), i \in\{0,1\}$, comprises precisely those $\boldsymbol{p} \in \mathrm{Cl}_{i}(\boldsymbol{V}, Q)$ which satisfy $\boldsymbol{p} \boldsymbol{q}=(-1)^{\partial \boldsymbol{p} \partial \boldsymbol{q}} \boldsymbol{q} \boldsymbol{p}$ for all homogeneous $\boldsymbol{q} \in \mathrm{Cl}(\boldsymbol{V}, Q)$; see [31, (3.5.2)] or [40, p. 152]. By (14), for all $\boldsymbol{p} \in \operatorname{Lip}^{\times}(\boldsymbol{V}, Q)$ and all vectors $\boldsymbol{x} \in \boldsymbol{V}$, we have

$$
\xi_{\boldsymbol{p}}(\boldsymbol{x})=\boldsymbol{p} \boldsymbol{x} \sigma(\boldsymbol{p})^{-1}=(-1)^{\partial \boldsymbol{p}} \boldsymbol{p} \boldsymbol{x} \boldsymbol{p}^{-1}=(-1)^{\partial \boldsymbol{p} \partial \boldsymbol{x}} \boldsymbol{p} \boldsymbol{x} \boldsymbol{p}^{-1} .
$$

Therefore, using that $\boldsymbol{V}$ generates $\mathrm{Cl}(\boldsymbol{V}, Q)$ as an algebra, we readily arrive at the intermediate result

$$
F^{\times} \subseteq \operatorname{ker} \xi=\operatorname{Lip}^{\times}(\boldsymbol{V}, Q) \cap Z^{g}(\mathrm{Cl}(\boldsymbol{V}, Q)) .
$$

From [31, (5.8.7) Lemma], the graded centre of $\mathrm{Cl}(\boldsymbol{V}, Q)$ equals the subalgebra generated by $\boldsymbol{V}^{\perp}$, which in turn may be viewed as $\mathrm{Cl}\left(\boldsymbol{V}^{\perp}, Q \mid \boldsymbol{V}^{\perp}\right)$. We therefore have

$$
F^{\times} \subseteq \operatorname{ker} \xi=\operatorname{Lip}^{\times}(\boldsymbol{V}, Q) \cap \operatorname{Cl}\left(\boldsymbol{V}^{\perp}, Q \mid \boldsymbol{V}^{\perp}\right) .
$$

The above description of the graded centre $Z^{g}(\mathrm{Cl}(\boldsymbol{V}, Q))$ as the subalgebra of $\mathrm{Cl}(\boldsymbol{V}, Q)$ generated by $\boldsymbol{V}^{\perp}$ can also be read off from [32, (1.8) a), (1.9) a)]. Likewise, the result about ker $\xi$ may easily be derived from [23, (22) Cor.] or [32, (2.2) Satz]. However, the author of [32] states some essential results without proof (just quoting his thesis). The corresponding proofs, despite their announcement in [32], never got published.

Below we collect a few more results, which are to be used later on.

Lemma 4.2. Let $(\boldsymbol{V}, Q)$ be a metric vector space. Then the kernel of the twisted adjoint representation $\xi$ of the Lipschitz group $\operatorname{Lip}^{\times}(\boldsymbol{V}, Q)$ satisfies the following properties.

(a) If $Q\left(\boldsymbol{V}^{\perp}\right)=\{0\}$, then $\operatorname{ker} \xi=\operatorname{ker}_{0} \xi$ and so $\operatorname{ker} \xi$ is a subgroup of $\operatorname{Lip}_{0}^{\times}(\boldsymbol{V}, Q)$.

(b) Given any regular vector $\boldsymbol{r} \in \boldsymbol{V}^{\perp}$ we have $\boldsymbol{r} \cdot\left(\operatorname{ker}_{0} \xi\right)=\operatorname{ker}_{1} \xi$. Therefore, whenever $Q\left(\boldsymbol{V}^{\perp}\right) \neq\{0\}$, $\operatorname{ker} \xi$ is not a subgroup of $\operatorname{Lip}_{0}^{\times}(\boldsymbol{V}, Q)$.

(c) If $\operatorname{dim} \boldsymbol{V}^{\perp} \leq 1$, then $\mathrm{ker}_{0} \xi=F^{\times}$. 
(d) If $\operatorname{dim} \boldsymbol{V}^{\perp} \geq 2$, then for any two-dimensional subspace $\boldsymbol{L} \subseteq \boldsymbol{V}^{\perp}$ there are linearly independent vectors $\boldsymbol{a}, \boldsymbol{b} \in \boldsymbol{L}$ such that

$$
\left\{x+y \boldsymbol{a} \boldsymbol{b} \mid x \in F^{\times}, y \in F\right\} \subseteq \operatorname{ker}_{0} \xi .
$$

Proof. (a) This is an immediate consequence of (5), applied to $\mathrm{Cl}\left(\boldsymbol{V}^{\perp}, Q \mid \boldsymbol{V}^{\perp}\right)$, and (15).

(b) By (15), $\boldsymbol{r} \in \boldsymbol{V}^{\perp}$ implies $\boldsymbol{r} \in \operatorname{ker} \xi$, which proves the assertions.

(c) We infer from $\operatorname{dim} \boldsymbol{V}^{\perp} \leq 1$ that $\mathrm{Cl}_{0}\left(\boldsymbol{V}^{\perp}, Q \mid \boldsymbol{V}^{\perp}\right)=F$. Hence (15) gives $F^{\times} \subseteq \operatorname{ker}_{0} \xi \subseteq \mathrm{Cl}_{0}^{\times}\left(\boldsymbol{V}^{\perp}, Q \mid \boldsymbol{V}^{\perp}\right)=F^{\times}$.

(d) We distinguish three cases: (i) $\boldsymbol{L}$ is totally singular; (ii) $\boldsymbol{L}$ contains no singular vectors; (iii) $\boldsymbol{L}$ contains a regular and a singular vector. In the first two cases we choose any linearly independent vectors $\boldsymbol{a}, \boldsymbol{b} \in \boldsymbol{L}$. If (iii) applies, we choose $\boldsymbol{a} \in \boldsymbol{L}$ regular and $\boldsymbol{b} \in \boldsymbol{L}$ singular. Now pick any element $x+y \boldsymbol{a} \boldsymbol{b}$ as appearing in (16). In view of (15), it suffices to verify that $x+y \boldsymbol{a} \boldsymbol{b} \in \operatorname{Lip}_{0}^{\times}(\boldsymbol{V}, Q)$. In case (i), this follows from

$$
x+y \boldsymbol{a} \boldsymbol{b}=x\left(1+\left(x^{-1} y \boldsymbol{a}\right) \boldsymbol{b}\right),
$$

$x \in F^{\times}$and $Q\left(x^{-1} y \boldsymbol{a}\right)=Q(\boldsymbol{b})=B\left(x^{-1} y \boldsymbol{a}, \boldsymbol{b}\right)=0$. Otherwise, $\boldsymbol{a}$ is regular and so Char $F=2$. By writing

$$
x+y \boldsymbol{a} \boldsymbol{b}=\boldsymbol{a}\left(x \boldsymbol{a}^{-1}+y \boldsymbol{b}\right),
$$

it remains to verify that $x \boldsymbol{a}^{-1}+y \boldsymbol{b}$ is regular. In case (ii), this turns out trivial. In case (iii), we have $Q\left(x \boldsymbol{a}^{-1}+y \boldsymbol{b}\right)=x^{2} Q\left(\boldsymbol{a}^{-1}\right)+0 \neq 0$.

\section{Projective Metric Geometry}

Let $\boldsymbol{V}$ be a vector space over $F$ as described at the beginning of Sect. 2. By the projective space $\mathbb{P}(\boldsymbol{V})$ we mean here the set of all subspaces of $\boldsymbol{V}$ with incidence being symmetrised inclusion [6, p. 30]. The dimension ${ }^{2}$ of $\mathbb{P}(\boldsymbol{V})$ is one less than the dimension of $\boldsymbol{V}$; that is, $\mathbb{P}(\boldsymbol{V})$ has projective dimension $n$. We adopt the usual geometric terms: points, lines and planes are the subspaces of $\boldsymbol{V}$ with (vector) dimension one, two, and three, respectively. Likewise, any subspace $\boldsymbol{T}$ of $\boldsymbol{V}$ gives rise to a projective space $\mathbb{P}(\boldsymbol{T})$, which is a substructure of $\mathbb{P}(\boldsymbol{V})$. The general linear group $\mathrm{GL}(\boldsymbol{V})$ acts in a canonical way on $\mathbb{P}(\boldsymbol{V})$ : any $\kappa \in \mathrm{GL}(\boldsymbol{V})$ determines a projective collineation on $\mathbb{P}(\boldsymbol{V})$, which is given by $\boldsymbol{X} \mapsto \kappa(\boldsymbol{X})$ for all $\boldsymbol{X} \in \mathbb{P}(\boldsymbol{V})$. The kernel of this action of $\mathrm{GL}(\boldsymbol{V})$ equals $F^{\times} \operatorname{id}_{V}$.

Next, assume $(\boldsymbol{V}, Q)$ to be a metric vector space. Then $Q$ can been used to furnish the projective space with "additional structure", thus making it into a projective metric space $\mathbb{P}(\boldsymbol{V}, Q)$. Thereby, for all $c \in F^{\times}$, the spaces $\mathbb{P}(\boldsymbol{V}, Q)$ and $\mathbb{P}(\boldsymbol{V}, c Q)$ are considered as being equal. We refer to [50] for a

\footnotetext{
${ }^{2}$ In order to avoid ambiguity, we shall frequently add the attribute "projective" when speaking about the dimension of a projective space.
} 
detailed description under the assumption $Q(\boldsymbol{V}) \neq\{0\}$; otherwise any "additional structure" arising from $Q$ is trivial. Let us recall a few notions derived from $(\boldsymbol{V}, Q)$ that remain unchanged if $Q$ is replaced by $c Q$. The orthogonality relations of $(\boldsymbol{V}, Q)$ and of $(\boldsymbol{V}, c Q)$ coincide. All points $F \boldsymbol{s}$ with $\boldsymbol{s} \in \boldsymbol{V}$ being singular constitute the absolute quadric $\mathcal{F}(\boldsymbol{V}, Q)$ of $\mathbb{P}(\boldsymbol{V}, Q) .{ }^{3}$ This quadric does not alter when going over to $c Q$. Also, we have $\operatorname{GO}(\boldsymbol{V}, Q)=\mathrm{GO}(\boldsymbol{V}, c Q)$, $\mathrm{O}(\boldsymbol{V}, Q)=\mathrm{O}(\boldsymbol{V}, c Q)$ and $\mathrm{O}^{\prime}(\boldsymbol{V}, Q)=\mathrm{O}^{\prime}(\boldsymbol{V}, c Q)$.

In contrast, the Clifford algebras $\mathrm{Cl}(\boldsymbol{V}, Q)$ and $\mathrm{Cl}(\boldsymbol{V}, c Q), c \in F^{\times}$, need not be isomorphic; see Example 6.1, where it is also shown that an analogous statement holds for the associated Lipschitz groups. Nevertheless, for the remaining part of this section, we shall make extensive use of the Clifford algebra $\mathrm{Cl}(\boldsymbol{V}, Q)$. The problem of how things change when going over to $\mathrm{Cl}(\boldsymbol{V}, c Q)$ will be addressed in Sect. 6 .

By the above, any isometry $\phi \in \mathrm{O}^{\prime}(\boldsymbol{V}, Q)$ determines a projective collineation of $\mathbb{P}(\boldsymbol{V}, Q)$. This action of $\mathrm{O}^{\prime}(\boldsymbol{V}, Q)$ on $\mathbb{P}(\boldsymbol{V}, Q)$ has the kernel

$$
\mathrm{I}^{\prime}(\boldsymbol{V}, Q):=\mathrm{O}^{\prime}(\boldsymbol{V}, Q) \cap\left\{\mathrm{id}_{\boldsymbol{V}},-\mathrm{id}_{\boldsymbol{V}}\right\} .
$$

The quotient of $\mathrm{O}^{\prime}(\boldsymbol{V}, Q)$ by $\mathrm{I}^{\prime}(\boldsymbol{V}, Q)$ is the projective weak orthogonal group, in symbols $\mathrm{PO}^{\prime}(\boldsymbol{V}, Q)$. Then

$$
\left|\mathrm{I}^{\prime}(\boldsymbol{V}, Q)\right|=1 \Leftrightarrow\left(\boldsymbol{V}=\{0\} \text { or } \boldsymbol{V}^{\perp} \neq\{0\} \text { or Char } F=2\right) .
$$

On the other hand, $\left|\mathrm{I}^{\prime}(\boldsymbol{V}, Q)\right| \neq 1$ implies $\left|\mathrm{I}^{\prime}(\boldsymbol{V}, Q)\right|=2$.

Now we change over to the projective space on the Clifford algebra $\mathrm{Cl}(\boldsymbol{V}, Q)$, where we introduce several point sets. Given such a set, say $\mathcal{S}$, we define $\mathcal{S}_{i}, i \in\{0,1\}$, to be the subset of $\mathcal{S}$ comprising all points that are contained in $\mathrm{Cl}_{i}(\boldsymbol{V}, Q)$. We start by defining

$$
\mathcal{H}(\boldsymbol{V}, Q):=\left\{F \boldsymbol{p} \mid \boldsymbol{p} \in \mathrm{Cl}_{0}^{\times}(\boldsymbol{V}, Q) \cup \mathrm{Cl}_{1}^{\times}(\boldsymbol{V}, Q)\right\}
$$

and proceed by making $\mathcal{H}(\boldsymbol{V}, Q)$ into a (multiplicative) group as follows: $(F \boldsymbol{p})(F \boldsymbol{q}):=F(\boldsymbol{p q})$ for all $F \boldsymbol{p}, F \boldsymbol{q} \in \mathcal{H}(\boldsymbol{V}, Q)$. Clearly, there is a canonical isomorphism of groups

$$
\left(\mathrm{Cl}_{0}^{\times}(\boldsymbol{V}, Q) \cup \mathrm{Cl}_{1}^{\times}(\boldsymbol{V}, Q)\right) / F^{\times} \rightarrow \mathcal{H}(\boldsymbol{V}, Q): F^{\times} \boldsymbol{p} \mapsto F \boldsymbol{p} .
$$

So, essentially, the two groups from above are the same. The Lipschitz monoid $\operatorname{Lip}(\boldsymbol{V}, Q)$ gives rise to the point set

$$
\mathcal{M}(\boldsymbol{V}, Q):=\{F \boldsymbol{p} \mid 0 \neq \boldsymbol{p} \in \operatorname{Lip}(\boldsymbol{V}, Q)\} .
$$

By the definition of $\operatorname{Lip}(\boldsymbol{V}, Q)$, the point set $\mathcal{M}(\boldsymbol{V}, Q)$ is the disjoint union of $\mathcal{M}_{0}(\boldsymbol{V}, Q)$ and $\mathcal{M}_{1}(\boldsymbol{V}, Q)$.

Remark 5.1. The sets $\mathcal{M}_{0}(\boldsymbol{V}, Q)$ and $\mathcal{M}_{1}(\boldsymbol{V}, Q)$ are algebraic varieties of the projective spaces on $\mathrm{Cl}_{0}(\boldsymbol{V}, Q)$ and $\mathrm{Cl}_{1}(\boldsymbol{V}, Q)$, respectively. See [25, p. 673] and [24], where a wealth of further properties of these varieties can be found. In particular, all subspaces of $\mathbb{P}(\mathrm{Cl}(\boldsymbol{V}, Q))$ whose point set is contained in

\footnotetext{
${ }^{3}$ Since we allow $Q$ to be the zero form, $\mathcal{F}(\boldsymbol{V}, Q)$ may coincide with the point set of $\mathbb{P}(\boldsymbol{V})$.
} 
$\mathcal{M}(\boldsymbol{V}, Q)$ have been determined there. Let us just mention the following particular case. If $\operatorname{dim} \boldsymbol{V} \leq 3$, then $\mathcal{M}_{0}(\boldsymbol{V}, Q)$ resp. $\mathcal{M}_{1}(\boldsymbol{V}, Q)$ comprises all points of $\mathbb{P}\left(\mathrm{Cl}_{0}(\boldsymbol{V}, Q)\right)$ resp. $\mathbb{P}\left(\mathrm{Cl}_{1}(\boldsymbol{V}, Q)\right)[23,(31)$ Lemma].

Remark 5.2. In general, the point set $\mathcal{M}(\boldsymbol{V}, Q)$ cannot be made into a monoid by following the path taken above. This is because the product of two non-zero elements of $\operatorname{Lip}(\boldsymbol{V}, Q)$ may be the zero vector, which fails to span a point.

Our third point set is

$$
\mathcal{G}(\boldsymbol{V}, Q):=\mathcal{H}(\boldsymbol{V}, Q) \cap \mathcal{M}(\boldsymbol{V}, Q)=\left\{F \boldsymbol{p} \mid \boldsymbol{p} \in \operatorname{Lip}^{\times}(\boldsymbol{V}, Q)\right\},
$$

which is a subgroup of $\mathcal{H}(\boldsymbol{V}, Q)$. The canonical isomorphism from (20) determines (by restriction) the isomorphism of groups

$$
\operatorname{Lip}^{\times}(\boldsymbol{V}, Q) / F^{\times} \rightarrow \mathcal{G}(\boldsymbol{V}, Q) .
$$

The Lipschitz group $\operatorname{Lip}^{\times}(\boldsymbol{V}, Q)$ contains $F^{\times}$as a normal subgroup. The representation (14) factors through the canonical homomorphism $\operatorname{Lip}^{\times}(\boldsymbol{V}, Q)$ $\rightarrow \operatorname{Lip}^{\times}(\boldsymbol{V}, Q) / F^{\times}$. We therefore have a surjective homomorphism of groups

$$
\operatorname{Lip}^{\times}(\boldsymbol{V}, Q) / F^{\times} \rightarrow \mathrm{O}^{\prime}(\boldsymbol{V}, Q): F^{\times} \boldsymbol{p} \mapsto \xi_{\boldsymbol{p}},
$$

the twisted adjoint representation of the quotient group $\operatorname{Lip}^{\times}(\boldsymbol{V}, Q) / F^{\times}$. By virtue of the inverse of (23), the twisted adjoint representation (24) and the canonical action of $\mathrm{O}^{\prime}(\boldsymbol{V}, Q)$ on $\mathbb{P}(\boldsymbol{V}, Q)$, the group $\mathcal{G}(\boldsymbol{V}, Q)$ as in (22) acts on the projective space $\mathbb{P}(\boldsymbol{V}, Q)$. Explicitly, for all $F \boldsymbol{p} \in \mathcal{G}(\boldsymbol{V}, Q)$ and all $\boldsymbol{X} \in \mathbb{P}(\boldsymbol{V}, Q)$, this action of $\mathcal{G}(\boldsymbol{V}, Q)$ takes the form

$$
F \boldsymbol{p} \mapsto\left(\boldsymbol{X} \mapsto \xi_{p}(\boldsymbol{X})=\boldsymbol{p} \boldsymbol{X} \sigma(\boldsymbol{p})^{-1}\right) .
$$

Furthermore, the action of $\mathcal{G}(\boldsymbol{V}, Q)$ on $\mathbb{P}(\boldsymbol{V}, Q)$ yields a surjective homomorphism of groups

$$
\theta: \mathcal{G}(\boldsymbol{V}, Q) \rightarrow \mathrm{PO}^{\prime}(\boldsymbol{V}, Q)=\mathrm{O}^{\prime}(\boldsymbol{V}, Q) / \mathrm{I}^{\prime}(\boldsymbol{V}, Q): F \boldsymbol{p} \mapsto \mathrm{I}^{\prime}(\boldsymbol{V}, Q) \circ \xi_{p} ;
$$

see (17). By our construction, $\operatorname{ker} \theta$ is just the kernel of the group action described in (25). This means

$$
\operatorname{ker} \theta=\left\{F \boldsymbol{p} \in \mathcal{G}(\boldsymbol{V}, Q) \mid \xi_{p} \in \mathrm{I}^{\prime}(\boldsymbol{V}, Q)\right\} .
$$

Remark 5.3. Let any $F \boldsymbol{m} \in \mathcal{H}(\boldsymbol{V}, Q)$ be given. The (group theoretic) left translation by $F \boldsymbol{m}$, that is the mapping $F \boldsymbol{q} \mapsto(F \boldsymbol{m})(F \boldsymbol{q})$ for all $F \boldsymbol{q} \in$ $\mathcal{H}(\boldsymbol{V}, Q)$, extends to a projective collineation of the ambient projective space. Obviously, the left translation $\lambda_{m} \in \mathrm{GL}(\mathrm{Cl}(\boldsymbol{V}, Q))$, which is given by $\boldsymbol{z} \mapsto \boldsymbol{m} \boldsymbol{z}$ for all $\boldsymbol{z} \in \mathrm{Cl}(\boldsymbol{V}, Q)$, provides a solution. The same properties hold, mutatis mutandis, for the right translation by $F \boldsymbol{m}$ and its counterpart $\rho_{\boldsymbol{m}}: \boldsymbol{z} \mapsto \boldsymbol{z} \boldsymbol{m}$ on the Clifford algebra.

Given any $F \boldsymbol{m} \in \mathcal{G}(\boldsymbol{V}, Q)$ the above observations clearly remain true when replacing $\mathcal{H}(\boldsymbol{V}, Q)$ with $\mathcal{G}(\boldsymbol{V}, Q)$. However, $\mathcal{G}(\boldsymbol{V}, Q)$ satisfies yet another property, which appears to be more substantial. The mapping that sends any $F \boldsymbol{q} \in \mathcal{G}(\boldsymbol{V}, Q)$ to its inverse $(F \boldsymbol{q})^{-1}$ also extends to a projective collineation of 
the ambient projective space. Such a collineation is determined by the reversal $\alpha$, since $\boldsymbol{q} \alpha(\boldsymbol{q}) \in F^{\times}$for all $\boldsymbol{q} \in \operatorname{Lip}^{\times}(\boldsymbol{V}, Q)$; see the noteworthy properties (i)-(iii) of the Lipschitz monoid mentioned at the beginning of Sect. 4 .

We proceed by examining in detail the kernel of the surjective homomorphism $\theta$ appearing in (26). We also investigate whether or not the subgroup $\theta\left(\mathcal{G}_{0}(\boldsymbol{V}, Q)\right)$ coincides with its ambient group $\mathrm{PO}^{\prime}(\boldsymbol{V}, Q)$. Clearly, whenever $\mathcal{G}_{0}(\boldsymbol{V}, Q)=\mathcal{G}(\boldsymbol{V}, Q)$, the answer to the latter question is affirmative. The large number of cases makes us split our findings into three theorems, according to the dimension of the radical $\boldsymbol{V}^{\perp}$.

Theorem 5.4. Let $(\boldsymbol{V}, Q)$ be a metric vector space with $\operatorname{dim} \boldsymbol{V}^{\perp}=0$. Then the surjective homomorphism $\theta: \mathcal{G}(\boldsymbol{V}, Q) \rightarrow \mathrm{PO}^{\prime}(\boldsymbol{V}, Q)$ has the following properties.

(a) If $\operatorname{dim} \boldsymbol{V}=0$, then $\operatorname{ker} \theta=\{F 1\}$ and $\mathcal{G}_{0}(\boldsymbol{V}, Q)=\mathcal{G}(\boldsymbol{V}, Q)$.

(b) If $\operatorname{dim} \boldsymbol{V}>0$ and Char $F=2$, then $\operatorname{ker} \theta=\{F 1\}$ and $\theta\left(\mathcal{G}_{0}(\boldsymbol{V}, Q)\right) \neq$ $\mathrm{PO}^{\prime}(\boldsymbol{V}, Q)$.

(c) If $\operatorname{dim} \boldsymbol{V}$ is odd and Char $F \neq 2$, then $\operatorname{ker} \theta$ comprises precisely two points, namely $F 1 \in \mathcal{G}_{0}(\boldsymbol{V}, Q)$ and one more point in $\mathcal{G}_{1}(\boldsymbol{V}, Q)$. Furthermore, $\theta\left(\mathcal{G}_{0}(\boldsymbol{V}, Q)\right)=\mathrm{PO}^{\prime}(\boldsymbol{V}, Q)$.

(d) If $\operatorname{dim} \boldsymbol{V}>0$ is even and Char $F \neq 2$, then $\operatorname{ker} \theta$ comprises precisely two points, both of which in $\mathcal{G}_{0}(\boldsymbol{V}, Q)$. Furthermore, $\theta\left(\mathcal{G}_{0}(\boldsymbol{V}, Q)\right) \neq \mathrm{PO}^{\prime}(\boldsymbol{V}, Q)$.

Proof. To begin with, we note that

$$
\operatorname{dim} \boldsymbol{V}^{\perp}=0 \text { implies } \operatorname{ker} \xi=\operatorname{ker}_{0} \xi=F^{\times},
$$

as follows readily from Lemma 4.2 (a) and (c).

(a) The assertions hold, since $\mathcal{G}(\boldsymbol{V}, Q)=\mathcal{G}_{0}(\boldsymbol{V}, Q)=\{F 1\}$.

(b) Due to Char $F=2$ and (18), we have $\mathrm{I}^{\prime}(\boldsymbol{V}, Q)=\left\{\operatorname{id}_{\boldsymbol{V}}\right\}$. Therefore (27) and (28) give $\operatorname{ker} \theta=\{F 1\}$. As $\boldsymbol{V}^{\perp}=\{0\}$ and $\operatorname{dim} \boldsymbol{V}>0$, there exists a regular vector $\boldsymbol{r} \in \boldsymbol{V}$ and so $F \boldsymbol{r} \in \mathcal{G}(\boldsymbol{V}, Q) \backslash \mathcal{G}_{0}(\boldsymbol{V}, Q)$. Since ker $\theta=\{F 1\}$ means that $\theta$ is injective, we obtain $\theta(F \boldsymbol{r}) \in \mathrm{PO}^{\prime}(\boldsymbol{V}, Q) \backslash \theta\left(\mathcal{G}_{0}(\boldsymbol{V}, Q)\right)$.

(c) Now (18) implies $\left|\mathrm{I}^{\prime}(\boldsymbol{V}, Q)\right|=2$. There exists an orthogonal basis $\left\{\boldsymbol{e}_{0}, \boldsymbol{e}_{1}\right.$, $\left.\ldots, \boldsymbol{e}_{n}\right\}$ of $(\boldsymbol{V}, Q)$ and we put

$$
e:=e_{0} e_{1} \cdots e_{n}
$$

From $\boldsymbol{V}^{\perp}=\{0\}$ we obtain $\boldsymbol{e} \in \operatorname{Lip}^{\times}(\boldsymbol{V}, Q)$. Together with $\operatorname{dim} \boldsymbol{V} \geq 1$ and Char $F \neq 2$ this shows $\xi_{\boldsymbol{e}}=-\mathrm{id}_{\boldsymbol{V}} \neq \mathrm{id}_{\boldsymbol{V}}$. So, from (27) and (28), $\operatorname{ker} \theta=\{F 1, F \boldsymbol{e}\}$ is a group of order two. Clearly, $F 1 \in \operatorname{ker}_{0} \theta$.

As $\operatorname{dim} \boldsymbol{V}$ is odd, $F \boldsymbol{e} \in \operatorname{ker}_{1} \theta$. We have

$$
\theta\left(\mathcal{G}_{0}(\boldsymbol{V}, Q)\right)=\theta\left(F \boldsymbol{e} \cdot \mathcal{G}_{0}(\boldsymbol{V}, Q)\right)=\theta\left(\mathcal{G}_{1}(\boldsymbol{V}, Q)\right),
$$

whence $\theta\left(\mathcal{G}_{0}(\boldsymbol{V}, Q)\right)=\theta(\mathcal{G}(\boldsymbol{V}, Q))=\operatorname{PO}^{\prime}(\boldsymbol{V}, Q)$. 
(d) We may repeat the reasoning from (c) up to the end of the first paragraph. By contrast, now $\operatorname{dim} \boldsymbol{V}>0$ is even, whence $F \boldsymbol{e} \in \operatorname{ker}_{0} \theta$. In analogy with (b), there is a regular vector $\boldsymbol{r} \in \boldsymbol{V}$ and so $F \boldsymbol{r} \in \mathcal{G}(\boldsymbol{V}, Q) \backslash \mathcal{G}_{0}(\boldsymbol{V}, Q)$. Taking into account that $\operatorname{ker} \theta$ is contained in $\mathcal{G}_{0}(\boldsymbol{V}, Q)$, we obtain $\theta(F \boldsymbol{r}) \in$ $\mathrm{PO}^{\prime}(\boldsymbol{V}, Q) \backslash \theta\left(\mathcal{G}_{0}(\boldsymbol{V}, Q)\right)$.

Note that under the hypotheses of Theorem 5.4 (b) the bilinear form $B$ is non-degenerate and alternating. Therefore, $\operatorname{dim} \boldsymbol{V}$ is necessarily even.

Theorem 5.5. Let $(\boldsymbol{V}, Q)$ be a metric vector space with $\operatorname{dim} \boldsymbol{V}^{\perp}=1$. Then the surjective homomorphism $\theta: \mathcal{G}(\boldsymbol{V}, Q) \rightarrow \mathrm{PO}^{\prime}(\boldsymbol{V}, Q)$ has the following properties.

(a) If $Q\left(\boldsymbol{V}^{\perp}\right)=\{0\}$, then $\operatorname{ker} \theta=\{F 1\}$.

(b) If $Q\left(\boldsymbol{V}^{\perp}\right)=\{0\}$ and $\operatorname{dim} \boldsymbol{V}=1$, then $\mathcal{G}_{0}(\boldsymbol{V}, Q)=\mathcal{G}(\boldsymbol{V}, Q)$.

(c) If $Q\left(\boldsymbol{V}^{\perp}\right)=\{0\}$ and $\operatorname{dim} \boldsymbol{V}>1$, then $\theta\left(\mathcal{G}_{0}(\boldsymbol{V}, Q)\right) \neq \mathrm{PO}^{\prime}(\boldsymbol{V}, Q)$.

(d) If $Q\left(\boldsymbol{V}^{\perp}\right) \neq\{0\}$, then $\operatorname{ker} \theta$ comprises precisely two points, namely $F 1 \in$ $\mathcal{G}_{0}(\boldsymbol{V}, Q)$ and one more point in $\mathcal{G}_{1}(\boldsymbol{V}, Q)$. Furthermore, $\theta\left(\mathcal{G}_{0}(\boldsymbol{V}, Q)\right)=$ $\mathrm{PO}^{\prime}(\boldsymbol{V}, Q)$.

Proof. First of all, from (18), we have that

$$
\operatorname{dim} \boldsymbol{V}^{\perp}=1 \text { implies } \mathrm{I}^{\prime}(\boldsymbol{V}, Q)=\left\{\operatorname{id}_{\boldsymbol{V}}\right\} .
$$

(a) From Lemma 4.2 (a) and (c), we have $\operatorname{ker} \xi=\operatorname{ker}_{0} \xi=F^{\times}$. Thus, using (27) and (31), we arrive at $\operatorname{ker} \theta=\{F 1\}$.

(b) By our hypotheses, we have $Q(\boldsymbol{V})=Q\left(\boldsymbol{V}^{\perp}\right)=\{0\}$. So (5) yields that $\mathrm{Cl}_{1}(\boldsymbol{V}, Q)$ contains no regular vector. Therefore $\mathcal{G}_{0}(\boldsymbol{V}, Q)=\mathcal{G}(\boldsymbol{V}, Q)$, as required.

(c) Due to $\boldsymbol{V}^{\perp} \neq \boldsymbol{V}$, there exists a regular vector in $\boldsymbol{r} \in \boldsymbol{V}$ and so $F \boldsymbol{r} \in$ $\mathcal{G}(\boldsymbol{V}, Q) \backslash \mathcal{G}_{0}(\boldsymbol{V}, Q)$. From (a), $\theta$ is injective, whence $\theta(F \boldsymbol{r}) \in \mathrm{PO}^{\prime}(\boldsymbol{V}, Q) \backslash \theta$ $\left(\mathcal{G}_{0}(\boldsymbol{V}, Q)\right)$.

(d) There is a regular vector $\boldsymbol{r} \in \boldsymbol{V}^{\perp}$. Using Lemma 4.2 (b) and (c), we obtain $^{4} \operatorname{ker} \xi=F^{\times} \cup F^{\times} \boldsymbol{r}$. Together with (27) and (31) this implies $\operatorname{ker} \theta=\{F 1, F \boldsymbol{r}\}$ and $F \boldsymbol{r} \cdot \mathcal{G}_{0}(\boldsymbol{V}, Q)=\mathcal{G}_{1}(\boldsymbol{V}, Q)$. Thus $\theta\left(\mathcal{G}_{0}(\boldsymbol{V}, Q)\right)=$ $\theta(\mathcal{G}(\boldsymbol{V}, Q))=\mathrm{PO}^{\prime}(\boldsymbol{V}, Q)$ follows (by replacing $\boldsymbol{e}$ with $\boldsymbol{r}$ ) in analogy to (30) .

Regarding Theorem $5.5(\mathrm{~d})$, it seems worth pointing out that $Q(\boldsymbol{V}) \neq\{0\}$ implies Char $F=2$. Together with $\operatorname{dim} \boldsymbol{V}^{\perp}=1$ this forces $\operatorname{dim} \boldsymbol{V}$ to be odd, since $B$ induces a non-degenerate alternating bilinear form on the quotient space $\boldsymbol{V} / \boldsymbol{V}^{\perp}$.

Theorem 5.6. Let $(\boldsymbol{V}, Q)$ be a metric vector space with $\operatorname{dim} \boldsymbol{V}^{\perp} \geq 2$. Then the surjective homomorphism $\theta: \mathcal{G}(\boldsymbol{V}, Q) \rightarrow \mathrm{PO}^{\prime}(\boldsymbol{V}, Q)$ has the following properties.

\footnotetext{
${ }^{4}$ We note that $F 1 \oplus F \boldsymbol{r}$ is a subalgebra of $\mathrm{Cl}\left(\boldsymbol{V}^{\perp}, Q \mid \boldsymbol{V}^{\perp}\right)$ and as such an inseparable quadratic extension field of $F$.
} 
(a) If $Q\left(\boldsymbol{V}^{\perp}\right)=\{0\}$, then $\operatorname{ker} \theta$ contains at least $|F|$ points from $\mathcal{G}_{0}(\boldsymbol{V}, Q)$ but no points from $\mathcal{G}_{1}(\boldsymbol{V}, Q)$.

(b) If $Q\left(\boldsymbol{V}^{\perp}\right)=\{0\}$ and $\operatorname{dim} \boldsymbol{V}=\operatorname{dim} \boldsymbol{V}^{\perp}$, then $\mathcal{G}_{0}(\boldsymbol{V}, Q)=\mathcal{G}(\boldsymbol{V}, Q)$.

(c) If $Q\left(\boldsymbol{V}^{\perp}\right)=\{0\}$ and $\operatorname{dim} \boldsymbol{V}>\operatorname{dim} \boldsymbol{V}^{\perp}$, then $\theta\left(\mathcal{G}_{0}(\boldsymbol{V}, Q)\right) \neq \mathrm{PO}^{\prime}(\boldsymbol{V}, Q)$.

(d) If $Q\left(\boldsymbol{V}^{\perp}\right) \neq\{0\}$, then $\operatorname{ker} \theta$ contains at least $|F|$ points from $\mathcal{G}_{0}(\boldsymbol{V}, Q)$ and at least $|F|$ points from $\mathcal{G}_{1}(\boldsymbol{V}, Q)$. Furthermore, $\theta\left(\mathcal{G}_{0}(\boldsymbol{V}, Q)\right)=$ $\mathrm{PO}^{\prime}(\boldsymbol{V}, Q)$.

Proof. (a) Lemma 4.2 (a) gives ker $\xi=\operatorname{ker}_{0} \xi$ and, from (18), we have $\mathrm{I}^{\prime}(\boldsymbol{V}, Q)$ $=\left\{\operatorname{id}_{\boldsymbol{V}}\right\}$. Thus (27) shows $\operatorname{ker} \theta=\operatorname{ker}_{0} \theta \subseteq \mathcal{G}_{0}(\boldsymbol{V}, Q)$. Let $\boldsymbol{L}$ be any two-dimensional subspace of $\boldsymbol{V}^{\perp}$. By adopting the terminology from Lemma $4.2(\mathrm{~d})$ and by substituting $x:=1$ in (16), we arrive at

$$
\{F(1+y \boldsymbol{a b}) \mid y \in F\} \subseteq \operatorname{ker}_{0} \theta \text { and }|\{F(1+y \boldsymbol{a} \boldsymbol{b}) \mid y \in F\}|=|F| .
$$

(b) The assertion follows from (5).

(c) From $\operatorname{dim} \boldsymbol{V}>\operatorname{dim} \boldsymbol{V}^{\perp}$, there exists a regular vector $\boldsymbol{r} \in \boldsymbol{V}$ and so $F \boldsymbol{r} \in \mathcal{G}(\boldsymbol{V}, Q) \backslash \mathcal{G}_{0}(\boldsymbol{V}, Q)$. In view of (a), $\operatorname{ker} \theta$ is contained in $\mathcal{G}_{0}(\boldsymbol{V}, Q)$. This in turn establishes $\theta(F \boldsymbol{r}) \in \mathrm{PO}^{\prime}(\boldsymbol{V}, Q) \backslash \theta\left(\mathcal{G}_{0}(\boldsymbol{V}, Q)\right)$.

(d) There exists a two-dimensional subspace $\boldsymbol{L}$ of $\boldsymbol{V}^{\perp}$ that contains a regular vector $\boldsymbol{a}$, say. We pick any vector $\boldsymbol{b} \in \boldsymbol{L}$ such that $\boldsymbol{a}, \boldsymbol{b}$ are linearly independent. According to the proof of Lemma 4.2 (d) we now use these vectors to obtain (16) and, as in (a), we substitute there $x:=1$. In this way we get a point set as in (32). This implies that $\operatorname{ker}_{1} \theta$ contains at least $|F|$ points, namely all points of the form $F(\boldsymbol{a}+y Q(\boldsymbol{a}) \boldsymbol{b})$ with $y$ varying in $F$.

Our description of $\operatorname{ker} \theta$ in Theorems 5.4, 5.5 and 5.6 improves [32, (2.3) Satz] in two ways: The result b) from there describes an analogue of our surjective homomorphism $\theta$ onto the group $\operatorname{PO}^{\prime}(\boldsymbol{V}, Q)$; however, it is based upon a subgroup of $\mathcal{H}(\boldsymbol{V}, Q)$ that in general is larger than our $\mathcal{G}(\boldsymbol{V}, Q)$. The result c) from there coincides with our findings whenever $\operatorname{Lip}^{\times}(\boldsymbol{V}, Q)$ is generated by all regular vectors of $(\boldsymbol{V}, Q)$, but provides no information about the exceptional cases (2) and (3).

Clearly, the surjective homomorphism $\theta$ as in (26) turns out to be an isomorphism of $\mathcal{G}(\boldsymbol{V}, Q)$ onto $\operatorname{PO}^{\prime}(\boldsymbol{V}, Q)$ if, and only if, $\operatorname{ker} \theta$ contains no point other than $F 1$. There are few possibilities for this to happen. All of them can be read off from Table 1. The first entry in each row (other than the header) provides a reference to the corresponding theorem, the remaining entries summarise the conditions that have to be met. Entries in braces are redundant and could be omitted.

Likewise, there is a rather small number of instances such that $\theta \mid \mathcal{G}_{0}(\boldsymbol{V}, Q)$ establishes an isomorphism of $\mathcal{G}_{0}(\boldsymbol{V}, Q)$ onto $\mathrm{PO}^{\prime}(\boldsymbol{V}, Q)$. An exhaustive summary is given in Table 2. Note that there is a single overlap between Tables 1 and 2. It pertains the trivial case $\operatorname{dim} \boldsymbol{V}=0$, where $\mathcal{G}(\boldsymbol{V}, Q)=\mathcal{G}_{0}(\boldsymbol{V}, Q)$. 
TABLE 1. $\mathcal{G}(\boldsymbol{V}, Q) \cong \mathrm{PO}^{\prime}(\boldsymbol{V}, Q)($ via $\theta)$

\begin{tabular}{lllll}
\hline Theorem & $\operatorname{dim} \boldsymbol{V}^{\perp}$ & $Q\left(\boldsymbol{V}^{\perp}\right)$ & $\operatorname{dim} \boldsymbol{V}$ & Char $F$ \\
\hline $5.4(\mathrm{a})$ & $(=0)$ & $(=\{0\})$ & $=0$ & \\
$5.4(\mathrm{~b})$ & $=0$ & $(=\{0\})$ & $>0$ (and even) & $=2$ \\
5.5 (a) & $=1$ & $=\{0\}$ & & \\
\hline
\end{tabular}

TABLE 2. $\mathcal{G}_{0}(\boldsymbol{V}, Q) \cong \mathrm{PO}^{\prime}(\boldsymbol{V}, Q)\left(\operatorname{via} \theta \mid \mathcal{G}_{0}(\boldsymbol{V}, Q)\right)$

\begin{tabular}{lllll}
\hline Theorem & $\operatorname{dim} \boldsymbol{V}^{\perp}$ & $Q\left(\boldsymbol{V}^{\perp}\right)$ & $\operatorname{dim} \boldsymbol{V}$ & Char $F$ \\
\hline 5.4 (a) & $(=0)$ & $(=\{0\})$ & $=0$ & \\
5.4 (c) & $=0$ & $(=\{0\})$ & odd & $\neq 2$ \\
5.5 (b) & $=1$ & $=\{0\}$ & $=1$ & \\
5.5 (d) & $=1$ & $\neq\{0\}$ & (odd) & $(=2)$ \\
\hline
\end{tabular}

TABLE 3. $\mathcal{G}(\boldsymbol{V}, Q) /\{F 1, F \boldsymbol{e}\} \cong \mathrm{PO}^{\prime}(\boldsymbol{V}, Q)$

\begin{tabular}{lllll}
\hline Theorem & $\operatorname{dim} \boldsymbol{V}^{\perp}$ & $Q\left(\boldsymbol{V}^{\perp}\right)$ & $\operatorname{dim} \boldsymbol{V}$ & Char $F$ \\
\hline $5.4(\mathrm{~d})$ & $=0$ & $(=\{0\})$ & $>0$ and even & $\neq 2$ \\
\hline
\end{tabular}

There is one more noteworthy situation, where $\theta$ fails to be injective, but $\operatorname{ker} \theta$ is a group of order two; the details are displayed in Table 3 . Here the group $\mathcal{G}_{0}(\boldsymbol{V}, Q)$ is equipped with the distinguished point $F \boldsymbol{e}$, which does not depend on the choice of the orthogonal basis $\left\{\boldsymbol{e}_{0}, \boldsymbol{e}_{1}, \ldots, \boldsymbol{e}_{n}\right\}$ of $\boldsymbol{V}$ that has been used in (29) when defining $\boldsymbol{e}$. The left translation $\lambda_{\boldsymbol{e}}$ (right translation $\left.\rho_{e}\right)$ acts on $\mathbb{P}(\mathrm{Cl}(\boldsymbol{V}, Q))$ as a projective collineation; see Remark 5.3. It is easy to verify that, for all $j \in\{0,1, \ldots, n\}$, we have $\boldsymbol{e}_{j}=-\boldsymbol{e}_{j} \boldsymbol{e}$. Using the basis (4) of $\mathrm{Cl}(\boldsymbol{V}, Q)$, we therefore obtain

$$
\lambda_{e}\left|\mathrm{Cl}_{0}(\boldsymbol{V}, Q)=\rho_{e}\right| \mathrm{Cl}_{0}(\boldsymbol{V}, Q) \text { and } \lambda_{e}\left|\mathrm{Cl}_{1}(\boldsymbol{V}, Q)=-\rho_{\boldsymbol{e}}\right| \mathrm{Cl}_{1}(\boldsymbol{V}, Q) .
$$

Thus, even though $\lambda_{e}$ and $\rho_{e}$ act differently on $\mathbb{P}(\mathrm{Cl}(\boldsymbol{V}, Q))$, their actions on $\mathbb{P}\left(\mathrm{Cl}_{0}(\boldsymbol{V}, Q)\right)$ and $\mathbb{P}\left(\mathrm{Cl}_{1}(\boldsymbol{V}, Q)\right)$ coincide.

To conclude this section, let us point out the following. If one of the situations from Table 1 occurs, then we may consider $\theta^{-1}$ as being a bijective "kinematic mapping" for the group $\operatorname{PO}^{\prime}(\boldsymbol{V}, Q)$. Note that this just a name for a series of examples rather than a general definition of such a mapping. Also, if one of the situations from Table 2 occurs, we have a bijective "kinematic mapping" for the group $\operatorname{PO}^{\prime}(\boldsymbol{V}, Q)$ given by $\left(\theta \mid \mathcal{G}_{0}(\boldsymbol{V}, Q)\right)^{-1}$. Under the restrictions of Table 3 we still have a kind of "kinematic mapping", but 
here one element of $\mathrm{PO}^{\prime}(\boldsymbol{V}, Q)$ is represented by an unordered pair of points from $\mathcal{G}(\boldsymbol{V}, Q)$. Some of the examples in [35, 3.4] and [36, Sect. 6] fit into the above concepts. However, the quoted works should be read with caution due to several misprints.

\section{A Comparison of Clifford Algebras}

We now switch back to a problem that we encountered in Sect. 5. Given a metric vector space $(\boldsymbol{V}, Q)$ and a constant $c \in F^{\times}$what is the relationship between the Clifford algebras $\mathrm{Cl}(\boldsymbol{V}, Q)$ and $\mathrm{Cl}(\boldsymbol{V}, c Q)$ ? For a closer look, we take into account that the identity id $\boldsymbol{V}$ is a similarity of ratio $c$ from $(\boldsymbol{V}, Q)$ onto $(\boldsymbol{V}, c Q)$. (Recall our convention that $c=1$ whenever $Q(\boldsymbol{V})=\{0\}$.) Therefore, according to (8), we obtain a linear bijection

$$
\mathrm{Cl}\left(\mathrm{id}_{\boldsymbol{V}}\right): \mathrm{Cl}(\boldsymbol{V}, Q) \rightarrow \mathrm{Cl}(\boldsymbol{V}, c Q) .
$$

This linear bijection allows us pulling back the algebra structure from $\mathrm{Cl}(\boldsymbol{V}, c Q)$ to $\mathrm{Cl}(\boldsymbol{V}, Q)$, which amounts to introducing a "new" multiplication $\odot_{c}$ on the vector space $\mathrm{Cl}(\boldsymbol{V}, Q)$. The algebra obtained in this way is isomorphic to $\mathrm{Cl}(\boldsymbol{V}, c Q)$ and will be abbreviated as $\mathrm{Cl}\left(\boldsymbol{V}, Q, \odot_{c}\right)$. A bridge between the initial and the new multiplication is provided by (6) and (7). We read off from there, for all $\boldsymbol{x}, \boldsymbol{y} \in \boldsymbol{V}$ and all $\boldsymbol{p} \in \mathrm{Cl}_{0}(\boldsymbol{V}, Q)$ :

$$
\boldsymbol{x} \boldsymbol{y}=c^{-1} \boldsymbol{x} \odot_{c} \boldsymbol{y}, \quad \boldsymbol{p} \boldsymbol{x}=\boldsymbol{p} \odot_{c} \boldsymbol{x}, \quad \boldsymbol{x} \boldsymbol{p}=\boldsymbol{x} \odot_{c} \boldsymbol{p} .
$$

Similarly, one may write up analogues of (9), (10), (11) and (12). In what follows right now, we shall adopt a slightly different point of view. We investigate the Clifford algebras of metric vector spaces $(\boldsymbol{V}, Q)$ and $(\tilde{\boldsymbol{V}}, \tilde{Q})$ admitting a similarity $\psi$ of ratio $c \in F^{\times}$with $\mathrm{Cl}(\psi)$ playing the role of the linear bijection (33). We shall return to $\mathrm{Cl}\left(\boldsymbol{V}, Q, \odot_{c}\right)$ only at the end of this section.

Example 6.1. Let $\boldsymbol{V}$ be a one-dimensional vector space over the field $\mathbb{R}$ of real numbers and let $\boldsymbol{i} \in \boldsymbol{V}$ be non-zero. We define a quadratic form $Q: \boldsymbol{V} \rightarrow$ $\mathbb{R}$ by $Q(\boldsymbol{i})=-1$. Then $\mathrm{Cl}(\boldsymbol{V}, Q)$ and the field $\mathbb{C}$ of complex numbers are isomorphic as $\mathbb{R}$-algebras, as follows from $\boldsymbol{i}^{2}=-1$. Furthermore, let $(\tilde{\boldsymbol{V}}, \tilde{Q})$ be isometric to $(\boldsymbol{V},-Q)$, whence there is a similarity $\psi: \boldsymbol{V} \rightarrow \tilde{\boldsymbol{V}}$ of ratio -1 . From $\tilde{Q}(\psi(\boldsymbol{i}))=\psi(\boldsymbol{i})^{2}=1$, the Clifford algebra $\mathrm{Cl}(\tilde{\boldsymbol{V}}, \tilde{Q})$ contains zero divisors ${ }^{5}$, whence the algebras $\operatorname{Cl}(\tilde{\boldsymbol{V}}, \tilde{Q})$ and $\mathrm{Cl}(\boldsymbol{V}, Q) \cong \mathbb{C}$ cannot be isomorphic; see also [43, Ex. 1.5, pp. 104-105] or the table of real Clifford algebras [43, p. 123]. The Lipschitz group $\operatorname{Lip}^{\times}(\boldsymbol{V}, Q)$ reads $\mathbb{R}^{\times} \cup \mathbb{R}^{\times} \boldsymbol{i}$. In $\operatorname{Lip}^{\times}(\boldsymbol{V}, Q)$ we have $\boldsymbol{i}^{2}=-1$, whereas no element of $\operatorname{Lip}^{\times}(\tilde{\boldsymbol{V}}, \tilde{Q})=\mathbb{R}^{\times} \cup \mathbb{R}^{\times} \psi(\boldsymbol{i})$ squares to -1 . So the Lipschitz groups of $(\boldsymbol{V}, Q)$ and $(\tilde{\boldsymbol{V}}, \tilde{Q})$ cannot be isomorphic either. In contrast, the quotient groups $\operatorname{Lip}^{\times}(\boldsymbol{V}, Q) / \mathbb{R}^{\times}$and $\operatorname{Lip}^{\times}(\tilde{\boldsymbol{V}}, \tilde{Q}) / \mathbb{R}^{\times}$both have order two and so they are isomorphic; see Theorem 6.3 .

\footnotetext{
${ }^{5}$ For example, $(1+\psi(i))(1-\psi(i))=0$.
} 
Theorem 6.2. Let $\psi: \boldsymbol{V} \rightarrow \tilde{\boldsymbol{V}}$ be a similarity of ratio $c \in F^{\times}$of metric vector spaces $(\boldsymbol{V}, Q)$ and $(\tilde{\boldsymbol{V}}, \tilde{Q})$. Then the Clifford extension $\mathrm{Cl}(\psi)$ has the following properties.

(a) $\mathrm{Cl}(\psi)$ maps the canonical filtration of $\mathrm{Cl}(\boldsymbol{V}, Q)$ onto the canonical filtration of $\mathrm{Cl}(\tilde{\boldsymbol{V}}, \tilde{Q})$.

(b) $\mathrm{Cl}(\psi)$ maps the group $\mathrm{Cl}_{0}^{\times}(\boldsymbol{V}, Q) \cup \mathrm{Cl}_{1}^{\times}(\boldsymbol{V}, Q)$ onto $\mathrm{Cl}_{0}^{\times}(\tilde{\boldsymbol{V}}, \tilde{Q}) \cup \mathrm{Cl}_{1}^{\times}(\tilde{\boldsymbol{V}}, \tilde{Q})$, the Lipschitz monoid $\operatorname{Lip}(\boldsymbol{V}, Q)$ onto $\operatorname{Lip}(\tilde{\boldsymbol{V}}, \tilde{Q})$ and, consequently, the Lipschitz group $\operatorname{Lip}^{\times}(\boldsymbol{V}, Q)$ onto $\operatorname{Lip}^{\times}(\tilde{\boldsymbol{V}}, \tilde{Q})$.

(c) For any $\boldsymbol{m} \in \operatorname{Lip}^{\times}(\boldsymbol{V}, Q)$, the isometries $\xi_{\boldsymbol{m}} \in \mathrm{O}^{\prime}(\boldsymbol{V}, Q)$ and $\tilde{\xi}_{\mathrm{Cl}(\psi)(\boldsymbol{m})} \in$ $\mathrm{O}^{\prime}(\tilde{\boldsymbol{V}}, \tilde{Q})$ satisfy $\psi \circ \xi_{\boldsymbol{m}}=\tilde{\xi}_{\mathrm{Cl}(\psi)(\boldsymbol{m})} \circ \psi$.

Proof. (a) Pick any integer $k \geq 0$. According to (10), $\mathrm{Cl}(\psi)$ takes any product of $k$ vectors from $\boldsymbol{V}$ to a product of $k$ vectors from $\tilde{\boldsymbol{V}}$, and an analogous statement holds for $\mathrm{Cl}\left(\psi^{-1}\right)=\mathrm{Cl}(\psi)^{-1}$. Thus the image of $\mathrm{Cl}^{\leq k}(\boldsymbol{V}, Q)$ under $\mathrm{Cl}(\psi)$ equals $\mathrm{Cl}^{\leq k}(\tilde{\boldsymbol{V}}, \tilde{Q})$, as required.

(b) To begin with, choose any $\boldsymbol{m} \in \mathrm{Cl}_{0}^{\times}(\boldsymbol{V}, Q) \cup \mathrm{Cl}_{1}^{\times}(\boldsymbol{V}, Q)$. From (12), the element $\mathrm{Cl}(\psi)(\boldsymbol{m})$ is in $\mathrm{Cl}_{0}^{\times}(\tilde{\boldsymbol{V}}, \tilde{Q}) \cup \mathrm{Cl}_{1}^{\times}(\tilde{\boldsymbol{V}}, \tilde{Q})$.

We now show that $\mathrm{Cl}(\psi)$ sends any generator of $\operatorname{Lip}(\boldsymbol{V}, Q)$, that is to mean any element $\boldsymbol{g}$ from $F, \boldsymbol{V}$ or the set (13), to a generator of $\operatorname{Lip}(\tilde{\boldsymbol{V}}, \tilde{Q})$ of the same kind. If $\boldsymbol{g}$ is in $F \cup \boldsymbol{V}$, then this is obvious. If $\boldsymbol{g}$ belongs to the set (13) or, more explicitly, if $\boldsymbol{g}=1+\boldsymbol{s} \boldsymbol{t}$ with $\boldsymbol{s}, \boldsymbol{t} \in \boldsymbol{V}$ subject to $Q(\boldsymbol{s})=Q(\boldsymbol{t})=B(\boldsymbol{s}, \boldsymbol{t})=0$, then $(9)$ implies $\mathrm{Cl}(\psi)(1+\boldsymbol{s} \boldsymbol{t})=$ $1+c^{-1} \psi(\boldsymbol{s}) \psi(\boldsymbol{t})$. As $\psi$ is a similarity, we obtain $\tilde{Q}\left(c^{-1} \psi(\boldsymbol{s})\right)=\tilde{Q}(\psi(\boldsymbol{t}))=$ $\tilde{B}\left(c^{-1} \psi(\boldsymbol{s}), \psi(\boldsymbol{t})\right)=0$, whence $\mathrm{Cl}(\psi)(\boldsymbol{g})$ has the required property.

Next, let any $\boldsymbol{m} \in \operatorname{Lip}(\boldsymbol{V}, Q)$ be given. By definition, $\boldsymbol{m}$ is a product of $k \geq 0$ generators $\boldsymbol{g}_{1}, \boldsymbol{g}_{2}, \ldots, \boldsymbol{g}_{k}$ that come from $F, \boldsymbol{V}$ or the set (13). From (10), there is an integer $q \geq 0$ such that

$$
\mathrm{Cl}(\psi)(\boldsymbol{m})=c^{-q} \mathrm{Cl}(\psi)\left(\boldsymbol{g}_{1}\right) \cdot \mathrm{Cl}(\psi)\left(\boldsymbol{g}_{2}\right) \cdots \mathrm{Cl}(\psi)\left(\boldsymbol{g}_{k}\right) .
$$

Thus, by the above, $\operatorname{Cl}(\psi)(\boldsymbol{m}) \in \operatorname{Lip}(\tilde{\boldsymbol{V}}, \tilde{Q})$.

Finally, (b) follows by repeating the above considerations with the similarity $\psi^{-1}$ instead of $\psi$.

(c) Choose any $\boldsymbol{x} \in \boldsymbol{V}$. Let $p$ be the number of factors with degree 1 in the product $\boldsymbol{m} \boldsymbol{x} \sigma(\boldsymbol{m})^{-1}$ and denote by $q$ the integer satisfying $2 q \leq p \leq$ $2 q+1$. As $\partial\left(\sigma(\boldsymbol{m})^{-1}\right)=\partial \boldsymbol{m}$, we may argue as follows. If $\partial \boldsymbol{m}=0$, then $p=1$ and $q=0$. If $\partial \boldsymbol{m}=1$, then $p=3$ and $q=1$. Therefore, we always have $q=\partial \boldsymbol{m}$. Now, from (10), (12) and $\mathrm{Cl}(\psi) \circ \sigma=\tilde{\sigma} \circ \mathrm{Cl}(\psi)$, we get

$$
\begin{aligned}
\psi\left(\xi_{\boldsymbol{m}}(\boldsymbol{x})\right) & =\mathrm{Cl}(\psi)\left(\boldsymbol{m} \boldsymbol{x} \sigma(\boldsymbol{m})^{-1}\right) \\
& =c^{-q} \mathrm{Cl}(\psi)(\boldsymbol{m}) \cdot \mathrm{Cl}(\psi)(\boldsymbol{x}) \cdot \mathrm{Cl}(\psi)\left(\sigma(\boldsymbol{m})^{-1}\right) \\
& =c^{-q+\partial \boldsymbol{m}} \mathrm{Cl}(\psi)(\boldsymbol{m}) \cdot \psi(\boldsymbol{x}) \cdot \tilde{\sigma}(\mathrm{Cl}(\psi)(\boldsymbol{m}))^{-1} \\
& =\tilde{\xi}_{\mathrm{Cl}(\psi)(\boldsymbol{m})}(\psi(\boldsymbol{x})),
\end{aligned}
$$


which completes the proof.

Theorem 6.3. Under the hypotheses of Theorem 6.2 the following hold.

(a) For any homogeneous element $\boldsymbol{m} \in \mathrm{Cl}(\boldsymbol{V}, Q)$ and all subspaces $\boldsymbol{H} \subseteq$ $\mathrm{Cl}_{0}(\boldsymbol{V}, Q) \cup \mathrm{Cl}_{1}(\boldsymbol{V}, Q)$, we have

$$
\begin{aligned}
& \mathrm{Cl}(\psi)(\boldsymbol{m H})=\mathrm{Cl}(\psi)(\boldsymbol{m}) \cdot \mathrm{Cl}(\psi)(\boldsymbol{H}), \\
& \mathrm{Cl}(\psi)(\boldsymbol{H} \boldsymbol{m})=\mathrm{Cl}(\psi)(\boldsymbol{H}) \cdot \mathrm{Cl}(\psi)(\boldsymbol{m}) .
\end{aligned}
$$

(b) The assignment $F^{\times} \boldsymbol{p} \mapsto F^{\times}(\mathrm{Cl}(\psi)(\boldsymbol{p}))=\mathrm{Cl}(\psi)\left(F^{\times} \boldsymbol{p}\right)$ defines an isomorphism of groups

$$
\left(\mathrm{Cl}_{0}^{\times}(\boldsymbol{V}, Q) \cup \mathrm{Cl}_{1}^{\times}(\boldsymbol{V}, Q)\right) / F^{\times} \rightarrow\left(\mathrm{Cl}_{0}^{\times}(\tilde{\boldsymbol{V}}, \tilde{Q}) \cup \mathrm{Cl}_{1}^{\times}(\tilde{\boldsymbol{V}}, \tilde{Q})\right) / F^{\times},
$$

an isomorphism of monoids $\operatorname{Lip}(\boldsymbol{V}, Q) / F^{\times} \rightarrow \operatorname{Lip}(\tilde{\boldsymbol{V}}, \tilde{Q}) / F^{\times}$and, consequently, also an isomorphism of groups

$$
\operatorname{Lip}^{\times}(\boldsymbol{V}, Q) / F^{\times} \rightarrow \operatorname{Lip}^{\times}(\tilde{\boldsymbol{V}}, \tilde{Q}) / F^{\times} .
$$

(c) The twisted adjoint representations of the quotient groups $\operatorname{Lip}^{\times}(\boldsymbol{V}, Q) / F^{\times}$ and $\operatorname{Lip}^{\times}(\tilde{\boldsymbol{V}}, \tilde{Q}) / F^{\times}$are equivalent by virtue of the isomorphism (36) and the given similarity $\psi: \boldsymbol{V} \rightarrow \tilde{\boldsymbol{V}}$.

Proof. The assertions are immediate from (9), (12) and Theorem 6.2.

Remark 6.4. Let us briefly sketch how to rephrase Theorem 6.3 (b) and (c) in terms of the projective spaces $\mathbb{P}(\mathrm{Cl}(\boldsymbol{V}, Q))$ and $\mathbb{P}(\mathrm{Cl}(\tilde{\boldsymbol{V}}, \tilde{Q}))$. The Clifford extension $\mathrm{Cl}(\psi)$ of the given similarity $\psi$ gives rise to a bijection $\mathcal{M}(\boldsymbol{V}, Q) \rightarrow$ $\mathcal{M}(\tilde{\boldsymbol{V}}, \tilde{Q})$ and it also yields an isomorphism linking the groups $\mathcal{H}(\boldsymbol{V}, Q)$ and $\mathcal{H}(\tilde{\boldsymbol{V}}, \tilde{Q})$. Consequently, it determines an isomorphism of the groups $\mathcal{G}(\boldsymbol{V}, Q)$ and $\mathcal{G}(\tilde{\boldsymbol{V}}, \tilde{Q})$ as well as their actions on $\mathbb{P}(\boldsymbol{V}, Q)$ and $\mathbb{P}(\tilde{\boldsymbol{V}}, \tilde{Q})$, respectively. Therefore, $\mathrm{Cl}(\psi)$ establishes also an isomorphism between the kernels of these group actions.

Remark 6.5. We still adhere to the hypotheses of Theorem 6.2. Moreover, we require $c$ to be a square in $F$. Upon choosing any square root of $c^{-1}$, say $\sqrt{c^{-1}}$, the following applies. The mapping $\omega:=\sqrt{c^{-1}} \psi$ is an isometry of $(\boldsymbol{V}, Q)$ onto $(\tilde{\boldsymbol{V}}, \tilde{Q})$. By the universal property of Clifford algebras, $\omega$ extends to a unique isomorphism of algebras $\mathrm{Cl}(\boldsymbol{V}, Q) \rightarrow \mathrm{Cl}(\tilde{\boldsymbol{V}}, \tilde{Q})$, which is easily seen to coincide with $\mathrm{Cl}(\omega)$. Also, we have $\mathrm{Cl}(\omega)=\mathrm{Cl}_{0}(\psi) \oplus \sqrt{c^{-1}} \mathrm{Cl}_{1}(\psi)$, whence the isomorphism $\mathrm{Cl}(\omega)$ allows for alternative proofs of our previous results. 6

We now switch back to our earlier point of view. Given $(\boldsymbol{V}, Q)$ and $c \in F^{\times}$ we consider $\mathrm{Cl}\left(\boldsymbol{V}, Q, \odot_{c}\right)$ as Clifford algebra of $(\boldsymbol{V}, c Q)$ with $\odot_{c}$ being subject to (34). From (8), the even subalgebras of $\mathrm{Cl}(\boldsymbol{V}, Q)$ and $\mathrm{Cl}\left(\boldsymbol{V}, Q, \odot_{c}\right)$ coincide

\footnotetext{
${ }^{6}$ If $c$ fails to be a square in $F$, then this can be carried out by going over to metric vector spaces over an appropriate quadratic extension of $F$.
} 
(as algebras), as do their odd parts (as vector spaces). Our quest for going over to the projective space on $\mathrm{Cl}(\boldsymbol{V}, Q)$ comes from an observation resulting from (35): for all homogeneous elements $\boldsymbol{p}, \boldsymbol{q} \in \mathrm{Cl}(\boldsymbol{V}, Q)$, we have $F(\boldsymbol{p q})=F\left(\boldsymbol{p} \odot_{c} \boldsymbol{q}\right)$ despite the fact that their products $\boldsymbol{p} \boldsymbol{q}$ and $\boldsymbol{p} \odot_{c} \boldsymbol{q}$ need not coincide. From (11), Theorems $6.2,6.3$ and Remark 6.4 we readily obtain:

Corollary 6.6. Let $(\boldsymbol{V}, Q)$ be a metric vector space and let $c \in F^{\times}$. The following notions arising from the Clifford algebra $\mathrm{Cl}(\boldsymbol{V}, Q)$ do not alter when going over to the algebra $\mathrm{Cl}\left(\boldsymbol{V}, Q, \odot_{c}\right)$ :

(a) The canonical filtration of $\mathrm{Cl}(\boldsymbol{V}, Q)$;

(b) for any homogeneous $\boldsymbol{m} \in \mathrm{Cl}^{\times}(\boldsymbol{V}, Q)$, the canonical action of the left translation $\lambda_{m}$ (right translation $\rho_{m}$ ) on the union of the projective spaces $\mathbb{P}\left(\mathrm{Cl}_{0}(\boldsymbol{V}, Q)\right)$ and $\mathbb{P}\left(\mathrm{Cl}_{1}(\boldsymbol{V}, Q)\right)$;

(c) the canonical action of the reversal $\alpha$ on the union of the projective spaces $\mathbb{P}\left(\mathrm{Cl}_{0}(\boldsymbol{V}, Q)\right)$ and $\mathbb{P}\left(\mathrm{Cl}_{1}(\boldsymbol{V}, Q)\right) ;$

(d) the group $\mathcal{H}(\boldsymbol{V}, Q) \cong\left(\mathrm{Cl}_{0}^{\times}(\boldsymbol{V}, Q) \cup \mathrm{Cl}_{1}^{\times}(\boldsymbol{V}, Q)\right) / F^{\times}$as in (19);

(e) the point set $\mathcal{M}(\boldsymbol{V}, Q)$ arising from the Lipschitz monoid $\operatorname{Lip}(\boldsymbol{V}, Q)$ according to $(21)$ and the group $\mathcal{G}(\boldsymbol{V}, Q) \cong \operatorname{Lip}^{\times}(\boldsymbol{V}, Q) / F^{\times}$as in $(22)$;

(f) the action of the group $\mathcal{G}(\boldsymbol{V}, Q)$ on the projective space $\mathbb{P}(\boldsymbol{V}, Q)$ as in (25).

\section{Future Research}

We are of the opinion that a closer look at low-dimensional examples should prove worthwhile. The first interesting class of examples are projective metric planes $(\operatorname{dim} \boldsymbol{V}=3)$, since they appear in the theory of absolute planes; see $[2,3]$, [34, Ch. III], [35, 3.4.1], [36, 6.1], [38,45], [50, 4.6] and the references therein; furthermore also finite Bolyai-Lobachevsky planes show up here $[41,42]$. In all these examples, the corresponding even Clifford algebra is a quaternion algebra $[17,54]$. In particular, an elliptic plane gives rise to a quaternion division algebra. Ultimately, one is lead to the following question: to which extent does the general theory of kinematic spaces (including the theory of Clifford parallelism) overlap with our findings as sketched in Remark 5.3. We refer, among others, to [5,22,33,47]. Going up one dimension $(\operatorname{dim} \boldsymbol{V}=4)$, one finds $\mathcal{M}_{0}(\boldsymbol{V}, Q)$ and $\mathcal{M}_{1}(\boldsymbol{V}, Q)$ as siblings of the classical Study quadric (see [52]) in a projective space of dimension 7; also here there are many results scattered over the literature; see [24, p. 463], [35, 3.4.2], [36, 6.2] and [51, Ch. 11].

Another step, still to be taken in a general context, is the inclusion of affine metric geometry. Over the real numbers this task has been accomplished quite a while ago and leads to what is called a homogeneous model. Related work can be read off from [35, 3.4.2], [36, 6.2], [20,21]. However, the approach used there relies on the signature of a real quadratic form, a notion which is no longer available over an arbitrary field. 
Last, but not least, also the general theory should allow for amplification. The results in [48], where points and planes of a three-dimensional projective space are used to represent motions of metric planes, suggest to investigate under which conditions the subspaces $\mathrm{Cl}_{0}(\boldsymbol{V}, Q)$ and $\mathrm{Cl}_{1}(\boldsymbol{V}, Q)$ of the Clifford algebra $\mathrm{Cl}(\boldsymbol{V}, Q)$ can be made into a dual pair of vector spaces in some meaningful way.

Funding Open access funding provided by TU Wien (TUW).

Availability of data and materials Not applicable.

\section{Declaration}

Conflict of interest The authors declare that they have no conflict of interest.

Code availability Not applicable.

Open Access. This article is licensed under a Creative Commons Attribution 4.0 International License, which permits use, sharing, adaptation, distribution and reproduction in any medium or format, as long as you give appropriate credit to the original author(s) and the source, provide a link to the Creative Commons licence, and indicate if changes were made. The images or other third party material in this article are included in the article's Creative Commons licence, unless indicated otherwise in a credit line to the material. If material is not included in the article's Creative Commons licence and your intended use is not permitted by statutory regulation or exceeds the permitted use, you will need to obtain permission directly from the copyright holder. To view a copy of this licence, visit http://creativecommons. org/licenses/by/4.0/.

\section{References}

[1] Atiyah, M.F., Bott, R., Shapiro, A.: Clifford modules. Topology 3, 3-38 (1964)

[2] Bachmann, F.: Aufbau der Geometrie aus dem Spiegelungsbegriff, volume 96 of Die Grundlehren der mathematischen Wissenschaften. Springer, Berlin (1973)

[3] Bachmann, F.: Ebene Spiegelungsgeometrie. Bibliographisches Institut, Mannheim (1989)

[4] Brady, N., McCammond, J.: Factoring Euclidean isometries. Int. J. Algebra Comput. 25, 325-347 (2015)

[5] Bröcker, L.: Kinematische Räume. Geom. Dedicata 1, 241-268 (1973)

[6] Buekenhout, F., Cameron, P.J.: Projective and affine geometry over division rings. In: Buekenhout, F. (ed.) Handbook of Incidence Geometry, pp. 27-62. North-Holland, Amsterdam (1995)

[7] Chevalley, C.: The Algebraic Theory of Spinors and Clifford Algebras. Springer, Berlin (1997)

[8] Cohn, P.M.: Basic Algebra. Springer, London (2003) 
[9] Ellers, E.W.: Decomposition of orthogonal, symplectic, and unitary isometries into simple isometries. Abh. Math. Sem. Univ. Hamburg 46, 97-127 (1977)

[10] Ellers, E.W.: Relations in classical groups. J. Algebra 51, 19-24 (1978)

[11] Ellers, E.W., Frank, R.: Products of quasireflections and transvections over local rings. J. Geom. 31, 69-78 (1988)

[12] Ellers, E.W., Frank, R., Nolte, W.: Bireflectionality of the weak orthogonal and the weak symplectic groups. J. Algebra 88, 63-67 (1984)

[13] Elman, R., Karpenko, N., Merkurjev, A.: The Algebraic and Geometric Theory of Quadratic Forms, volume 56 of American Mathematical Society Colloquium Publications. American Mathematical Society, Providence (2008)

[14] Frank, R., Mäurer, H.: Generators for the motion group of metric vector spaces. Geom. Dedicata 22, 225-233 (1987)

[15] Götzky, M.: Über die Erzeugenden der engeren unitären Gruppen. Arch. Math. (Basel) 19, 383-389 (1968)

[16] Götzky, M.: Unverkürzbare Produkte und Relationen in unitären Gruppen. Math. Z. 104, 1-15 (1968)

[17] Gross, B.H., Lucianovic, M.W.: On cubic rings and quaternion rings. J. Number Theory 129, 1468-1478 (2009)

[18] Grove, L.C.: Classical Groups and Geometric Algebra, volume 39 of Graduate Studies in Mathematics. American Mathematical Society, Providence (2002)

[19] Gunn, C.: Geometry, Kinematics, and Rigid Body Mechanics in Cayley-Klein Geometries. Technische Universität Berlin, Thesis (2011)

[20] Gunn, C.: Geometric algebras for Euclidean geometry. Adv. Appl. Clifford Algebras 27, 185-208 (2017)

[21] Gunn, C.G.: Doing Euclidean plane geometry using projective geometric algebra. Adv. Appl. Clifford Algebras 27, 1203-1232 (2017)

[22] Havlicek, H., Pasotti, S., Pianta, S.: Characterising Clifford parallelisms among Clifford-like parallelisms. Beitr. Algebra Geom. 62, 37-54 (2021)

[23] Helmstetter, J.: Lipschitz monoids and Vahlen matrices. Adv. Appl. Clifford Algebras 15, 83-122 (2005)

[24] Helmstetter, J.: Lipschitzian subspaces in Clifford algebras. J. Algebra 328, 461483 (2011)

[25] Helmstetter, J.: A survey of Lipschitz monoids. Adv. Appl. Clifford Algebras. 22, 665-688 (2012)

[26] Helmstetter, J.: Minimal algorithms for Lipschitz monoids and Vahlen monoids. J. Math. Res. 5, 39-51 (2013)

[27] Helmstetter, J.: Factorization of Lipschitzian elements. Adv. Appl. Clifford Algebras 24, 675-712 (2014)

[28] Helmstetter, J.: Conformal groups and Vahlen matrices. Adv. Appl. Clifford Algebras 27, 33-44 (2017)

[29] Helmstetter, J.: Products of reflections and triangularization of bilinear forms. J. Math. Res. 9, 18-31 (2017)

[30] Helmstetter, J.: Bilinear forms derived from Lipschitzian elements in Clifford algebras. Adv. Appl. Clifford Algebras 28, Article 25 (2018) 
[31] Helmstetter, J., Micali, A.: Quadratic Mappings and Clifford Algebras. Birkhäuser Verlag, Basel (2008)

[32] Jurk, R.: Zur Darstellung der klassischen Gruppen durch Clifford-Algebren. J. Geom. 16, 72-82 (1981)

[33] Karzel, H.: Kinematic spaces. In: Symposia Mathematica, Vol. XI (Convegno di Geometria, INDAM, Roma, 1972), pp. 413-439. Academic Press, London (1973)

[34] Karzel, H., Kroll, H.-J.: Geschichte der Geometrie seit Hilbert. Wissenschaftliche Buchgesellschaft, Darmstadt (1988)

[35] Klawitter, D.: Clifford Algebras. Springer Spektrum, Wiesbaden (2015)

[36] Klawitter, D., Hagemann, M.: Kinematic mappings for Cayley-Klein geometries via Clifford algebras. Beitr. Algebra Geom. 54, 737-761 (2013)

[37] Klopsch, P.: Gegen Loten abgeschlossene Untergruppen metrischer Vektorräume. J. Geom. 10, 69-84 (1977)

[38] Klopsch, P.: Algebraische Kennzeichnung angeordneter Bachmann-Räume. Geom. Dedicata 18, 249-259 (1985)

[39] Kneser, M.: Witts Satz über quadratische Formen und die Erzeugung orthogonaler Gruppen durch Spiegelungen. Math. Phys. Semesterber. 17, 33-45 (1970)

[40] Knus, M.-A.: Quadratic and Hermitian Forms Over Rings, volume 294 of Grundlehren der Mathematischen Wissenschaften. Springer, Berlin (1991)

[41] Korchmáros, G., Sonnino, A.: Finite Bolyai-Lobachevskii planes. Acta Math. Hung. 134, 405-415 (2012)

[42] Korchmáros, G., Sonnino, A.: Old and recent results on finite BolyaiLobachevsky planes. Mathematica 56(79), 59-73 (2014)

[43] Lam, T.Y.: Introduction to Quadratic Forms over Fields, volume 67 of Graduate Studies in Mathematics. American Mathematical Society, Providence (2005)

[44] Lounesto, P.: Clifford Algebras and Spinors, volume 286 of London Mathematical Society Lecture Note Series. Cambridge University Press, Cambridge (2001)

[45] Molnár, E.: Absolute circle (sphere) geometry by reflection. South Bohem. Math. Lett. 26, 45-61 (2018)

[46] Nolte, W.: Relationen zwischen einfachen Isometrien in orthogonalen Gruppen. In: Arnold, H.J., Benz, W., Wefelscheid, H. (eds.) Beiträge zur geometrischen Algebra (Proc. Sympos., Duisburg, 1976), volume 21 of Lehrbücher u. Monographien aus dem Gebiete der Exakt. Wiss., Math. Reihe, pp. 275-278 (1977)

[47] Pasotti, S.: Regular parallelisms in kinematic spaces. Discrete Math. 310, 31203125 (2010)

[48] Schröder, E.M.: Kennzeichnung und Darstellung kinematischer Räume metrischer Ebenen. Abh. Math. Sem. Univ. Hamburg 39, 184-230 (1973)

[49] Schröder, E.M.: Metrische Geometrie und Clifford-Algebren. Mitt. Math. Ges. Hamburg 11, 391-413 (1987)

[50] Schröder, E.M.: Metric geometry. In: Buekenhout, F. (ed.) Handbook of Incidence Geometry, pp. 945-1013. North-Holland, Amsterdam (1995)

[51] Selig, J.M.: Geometric Fundamentals of Robotics. Springer, New York (2005)

[52] Study, E.: Grundlagen und Ziele der analytischen Kinematik. Sitzungsber. Berl. Math. Ges. 12, 36-60 (1913) (erratum ibid. 94) 
[53] Taylor, D.E.: The Geometry of the Classical Groups, volume 9 of Sigma Series in Pure Mathematics. Heldermann, Berlin (1992)

[54] Voight, J.: Characterizing quaternion rings over an arbitrary base. J. Reine Angew. Math. 657, 113-134 (2011)

Hans Havlicek

Institut für Diskrete Mathematik und Geometrie

Technische Universität Wien

Wiedner Hauptstraße 8-10/104

1040 Wien

Austria

e-mail: havlicek@geometrie.tuwien.ac.at

Received: March 18, 2021.

Accepted: September 9, 2021.

Publisher's Note Springer Nature remains neutral with regard to jurisdictional claims in published maps and institutional affiliations. 